\title{
Mesnil-Esnard « Les Hautes Haies » (Seine- Maritime), une occupation du IIIe millénaire av. J.-
} C.

Mesnil-Esnard "Les Hautes Haies" (Seine-Maritime, France), a site of the 3th millenium $B C$

Mesnil-Esnard « Les Hautes-Haies » (Seine-Maritime), una ocupación del tercer milenario antes de J.-C.

Nicolas Fromont, Laurent Juhel, Jean-Yves Noël et Bruno Aubry

\section{OpenEdition}

Journals

Édition électronique

URL : https://journals.openedition.org/rao/2057

DOI : $10.4000 /$ rao. 2057

ISBN : 978-2-7535-3432-2

ISSN : $1775-3732$

Éditeur

Presses universitaires de Rennes

\section{Édition imprimée}

Date de publication : 25 décembre 2013

Pagination : $57-86$

ISBN : 978-2-7535-3430-8

ISSN : 0767-709X

Référence électronique

Nicolas Fromont, Laurent Juhel, Jean-Yves Noël et Bruno Aubry, « Mesnil-Esnard « Les Hautes Haies » (Seine-Maritime), une occupation du Ille millénaire av. J.-C. », Revue archéologique de l'Ouest [En ligne], 30 | 2013, mis en ligne le 25 décembre 2015, consulté le 22 août 2022. URL : http:// journals.openedition.org/rao/2057 ; DOI : https://doi.org/10.4000/rao.2057

Ce document a été généré automatiquement le 22 août 2022.

Tous droits réservés 


\title{
Mesnil-Esnard « Les Hautes Haies » (Seine-Maritime), une occupation du IIIe millénaire av. J.-C.
}

\author{
Mesnil-Esnard "Les Hautes Haies" (Seine-Maritime, France), a site of the 3th \\ millenium $B C$ \\ Mesnil-Esnard « Les Hautes-Haies » (Seine-Maritime), una ocupación del tercer \\ milenario antes de J.-C.
}

Nicolas Fromont, Laurent Juhel, Jean-Yves Noël et Bruno Aubry

1 Exploré par une équipe de l'Inrap en 2005, le site du Mesnil-Esnard «Les Hautes Haies » est situé en périphérie de Rouen (Seine-Maritime). Il a livré une importante série lithique attribuable à la fin du III millénaire av J.-C. (Aubry et al., 2004 ; Fromont et al., 2006). Les vestiges immobiliers ne sont représentés que par de rares structures. Le mobilier qui en provient est relativement modeste et ne permet aucune datation précise. En revanche, plus de 1600 éléments en silex sont éparpillés sous la forme d'une vaste nappe conservée sous le labour.

2 L'étude présentée met l'accent sur l'importance de l'analyse de l'industrie lithique pour appréhender ce type de gisement. Malgré les limites inhérentes à l'étude typologique, une datation de la série peut être proposée. Sur le plan fonctionnel, la faible densité et la nature peu variée des vestiges suggèrent que cette nappe constitue le reliquat d'une occupation dont la vocation domestique n'est pas assurée. Elle pourrait alors s'intégrer dans une unité plus vaste de type habitat ou village. À moins qu'elle ne constitue une " annexe » au sein du terroir exploité par ces unités domestiques. Face à ces questions, la lecture macroscopique de l'outillage en silex a trouvé ses limites. Elle justifie la pertinence d'une analyse tracéologique à fort grossissement, pour mieux cerner, à l'avenir, ce type de gisement. 


\section{État des connaissances : la fin du Néolithique et le début des âges des Métaux à l'échelle régionale}

3 La documentation sur la fin du Néolithique et le début de l'âge du Bronze est plutôt indigente et disparate en Haute-Normandie comme plus largement dans le Bassin parisien (Salanova et al., 2003). Cependant, la définition des groupes culturels qui se sont succédé ou côtoyés durant cette période repose sur diverses fouilles récentes qui apportent des éléments importants dans le domaine de l'habitat, du funéraire et de la culture matérielle (Marcigny et al., 2002; Martin et al., 1996). Ils complètent les diagnoses des ensembles culturels qui reposent encore trop souvent sur quelques éléments céramiques (Brunet et al., 2004).

4 Le Néolithique final pré-campaniforme (Salanova et Van der Linden, 2004) serait représenté par les ensembles $\mathrm{n}^{\circ} 3$ et 17 de Saint-Vigor-d'Ymonville « Les Sapinettes/La Mare des Mares » (Marcigny et al., 2002). Les ensembles n 3 et 4 de Poses « Le Vivier/Le Clos Saint-Quentin » sont aussi attribués à cette tranche chronologique et les influences du groupe de Gord y sont perceptibles, de même que celles de l'Artenac ou du Conguel (Billard et al., 1994).

5 Le Néolithique final campaniforme, caractérisé par diverses influences (bretonnes, anglaises, rhénanes...), s'illustre par les fameux gobelets campaniformes. En association, se rencontre parfois une céramique dite d'accompagnement résultant d'une fusion entre les productions $d u$ Néolithique final et des caractères campaniformes (Salanova, 2000 ; Strahm, 2004). Pour la Haute-Normandie, certaines découvertes donnent un éclairage ponctuel sur ce "phénomène campaniforme": Grossœuvre «Viancourt » (Billard et Paez-Rezende, 2000), Guerny « Le Bois Madame » (Martinez et al., 1999), Yport « Saint-Léonard » (Watté, 1992), Saint-Vigor-d'Ymonville "Les Sapinettes/La Mare des Mares", ensembles 25 à 40 (Marcigny et al., 2002). Il intervient à cheval sur la fin du Néolithique final et le début du Bronze ancien selon des modalités et des manifestations variables d'une région à l'autre (Salanova, 2000). De fait, la transition vers l'âge du Bronze et la genèse de celui-ci sont mal appréhendées. Parallèlement, sous les mêmes influences, apparaîtrait le Groupe des urnes à décor plastique, qui rassemble des formes céramiques plus ou moins similaires retrouvées sur tout le Nord-Ouest de l'Europe. Il est maintenant confondu dans une vaste entité culturelle dénommée " Manche - Mer-du-Nord » (Marcigny et al., 2005) caractérisée par des liens très forts entre le continent et l'Angleterre. Pour la fin du Bronze ancien, ce sont les vestiges recueillis à Saint-Vigor-d'Ymonville «Les Sapinettes/La Mare des Mares », ensembles 2, 2bis, 8, 9, 14 et 16 (Marcigny et al., 2002), qui donnent sur le plan régional un éclairage nouveau. L'aménagement de l'espace et le mobilier céramique de ce site trahissent des affinités avec ce qui est connu en France et en Angleterre sur des sites de tradition Deverel-Rimbury. Serait ainsi illustré un stade final du Bronze ancien qui pourrait être confondu avec le Groupe des urnes à décor plastique simple ou avec la phase I du groupe d'Eramecourt (op. cit.; Billard et al., 1996).

En Haute-Normandie, à l'horizon du III millénaire, la caractérisation de l'industrie lithique est particulièrement complexe, eu égard à différents paramètres. En premier lieu, la documentation souffre de l'absence d'ensembles clos ou, tout du moins, véritablement cohérents sur le plan chrono-stratigraphique. En effet, trop souvent, les séries disponibles sont tributaires de mélanges plus ou moins explicites avec d'autres horizons chronologiques. Cette difficulté est d'autant plus délicate à contourner que le 
mobilier lithique n'est pas forcément très caractéristique sur le plan technologique et typologique. Le problème de la représentativité des corpus est également posé puisque les surfaces fouillées sont généralement faibles.

7 Le modèle actuellement établi laisse pressentir une évolution progressive de la technologie du débitage au cours du III ${ }^{\mathrm{e}}$ millénaire (Billard et al., 1994 ; Martinez et al., 1999 ; Prost, 1999 ; Martial, 1995). La fin de la prédétermination du débitage d'éclats et surtout de lames, caractérisant les périodes ancienne et moyenne du Néolithique, aboutit aux schémas opératoires très simplifiés et opportunistes de l'âge du Bronze. Néanmoins, ce modèle est sans doute plus complexe. À titre d'exemple, les ensembles $\mathrm{n}^{\circ} 4$ à 7 de Poses «Le Vivier/Le Clos Saint-Quentin» (Billard et al., 1994), échelonnés entre le Néolithique final et le Bronze ancien, ne montrent pas d'évolution technologique significative. Le débitage de l'ensemble $\mathrm{n}^{\circ} 7$, le plus tardif, présente une plus grande préparation des plans de frappe en comparaison avec les assemblages plus anciens (Billard et al., 1994). Le choix des matières premières exploitées semble, dans certains cas, conditionner le débitage, comme l'illustre certainement le cas du débitage sur enclume (Billard et al., 1991).

8 Les changements qui concernent l'outillage sont en revanche plus significatifs (Martial 1995 ; Ghesquière et al., 1997 ; Prost, 1999). Au Néolithique récent et final, on note la perduration d'un fonds hérité du Néolithique moyen. Parmi celui-ci, l'outillage retouché tend à diminuer au cours du III millénaire, au profit d'outils a posteriori qui composent à l'âge du Bronze la majeure partie de l'outillage.

Ce modèle cohérent reste toutefois établi sur des bases statistiques largement tributaires de la représentativité des assemblages considérés et du panel, plus ou moins constant, des activités où il intervient sur le site considéré. Certains éléments spécifiques permettent a priori la distinction entre les assemblages du Néolithique récent/final et ceux de l'âge du Bronze. Il s'agit notamment des racloirs à encoche et des microdenticulés dont les proportions s'infléchiraient significativement, voire disparaîtraient totalement, dès le Bronze ancien.

Cette évolution des assemblages lithiques au cours du III ${ }^{\mathrm{e}}$ millénaire reste malgré tout délicate à illustrer en Haute-Normandie, eu égard à la faiblesse de la documentation disponible. Cette industrie demande donc à être mieux caractérisée par l'étude d'assemblages abondants et/ou stratigraphiquement cohérents. Le corpus du MesnilEsnard apparaît à ce titre pertinent, même s'il soulève à nouveau nombre de questions inhérentes aux séries régionales de cette période.

\section{Présentation générale du site}

\section{Contexte environnemental}

11 L'occupation reconnue est implantée sur la commune du Mesnil-Esnard dans la banlieue de Rouen (fig. 1). La configuration topographique est celle d'un vaste éperon correspondant à la terminaison du plateau crayeux de Boos entre les vallées de la Seine, à l'ouest, et celle de l'Aubette, au nord. Vers la Seine, la rupture du plateau est brutale. Les falaises livrent les formations carbonatées à silex du Crétacé (Coguel et Sangnier, 1967a et b). À l'opposé, au nord, la pente est plus douce car ces mêmes formations sont recouvertes de colluvions. 
Les vestiges fouillés se rencontrent sur le plateau, à une altitude d'environ 155 mètres, à l'amorce d'un petit vallon sec, la Valette de la Lande, qui se dirige vers l'Aubette. Le substrat crétacé est ici recouvert d'argile à silex et d'une couverture de limon éolien.

Figure 1 : Situation topographique du site des « Hautes Haies » au Mesnil-Esnard.

Figure 1 : Location of "Les Hautes Haies", Mesnil-Esnard.

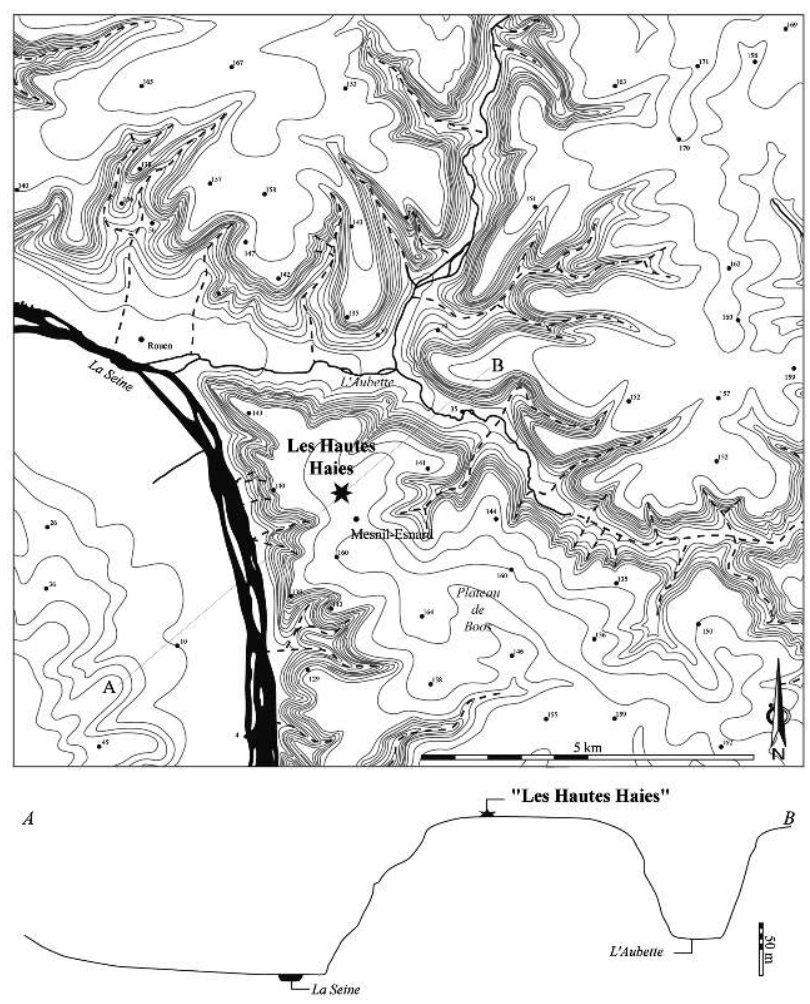

DAO N. Fromont.

\section{Contexte stratigraphique du mobilier}

13 La partie supérieure du sol actuel est constituée de limon remanié par les labours sur une épaisseur excédant rarement trente centimètres (fig. 2). En dessous, l'horizon A du sol holocène est préservée sur trente à cinquante centimètres. Il s'agit d'un limon brungris plus ou moins bioturbé et chargé en matière organique. Localement, sous la base des labours et sur une épaisseur de quelques centimètres, des phénomènes d'hydromorphie affectent surtout une dépression topographique au centre de l'emprise. Sans solution de continuité avec l'horizon A, on passe à un limon argileux orangé composant l'horizon Bt. Les traces de bioturbation y sont rares, alors que des phénomènes d'hydromorphie s'y retrouvent. Localement, une faible charge en cailloutis (silex patinés et émoussés centimétriques) s'ajoute au limon argileux. Enfin, après une transition plus ou moins longue, apparaît le lœss (horizon C). 
Figure 2 : Logs stratigraphiques des horizons superficiels rencontrés sur le site du Mesnil-Esnard "Les Hautes Haies » et dispersion verticale des vestiges de la nappe (1) apparaissant en grisé sur le plan (2).

Figure 2 : Stratigraphy of the superficial formations, vertical (1) and horizontal dispersal of vestiges (2).

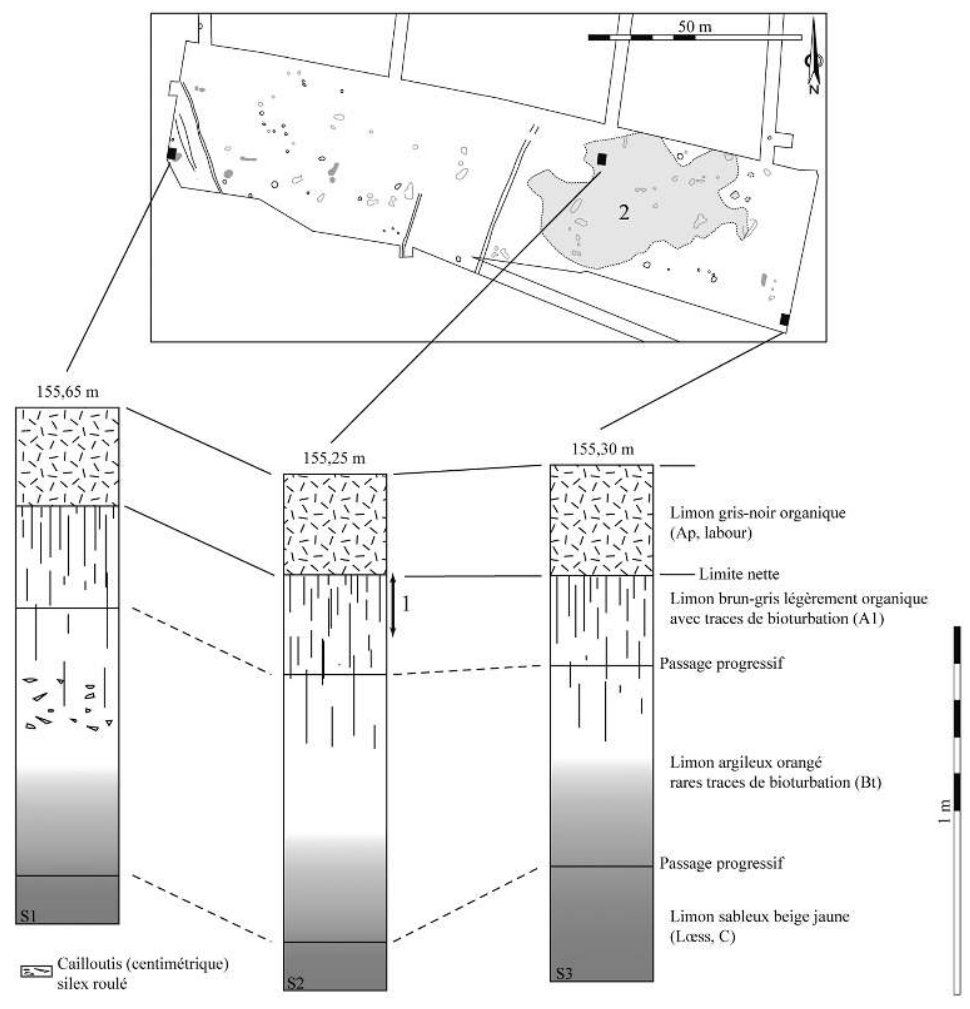

DAO N. Fromont.

La puissance des horizons A et Bt subit de faibles variations puisque le toit du lœss se trouve entre quatre-vingt-dix et cent vingt centimètres par rapport à la surface actuelle. En conséquence, l'érosion du sol après l'occupation néolithique semble très réduite, certainement localisée et/ou générée au niveau de certains microreliefs et de certaines installations anthropiques. Dès lors, il n'est pas aisé d'isoler le rôle de l'érosion dans la conservation, par recouvrement, ou la destruction, par troncature, des vestiges archéologiques. Le niveau de sol fréquenté par les Néolithiques est donc certainement sensiblement équivalent à celui d'aujourd'hui. Ainsi, la pédogenèse, et dans une moindre mesure les labours, nous privent-ils de tout ou partie des aménagements anciens susceptibles d'affecter les soixante premiers centimètres du sol. Le peu de structures observées dans l'espace de l'emprise, malgré la présence d'une vaste nappe de mobilier, peut trouver ici une explication non exclusive. L'essentiel des vestiges immobiliers n'est clairement perceptible qu'au niveau de la transition entre les horizons $\mathrm{A}$ et $\mathrm{Bt}$. Leur apparition plus en hauteur est difficile à déceler, sauf si leur remplissage contient des éléments remarquables (blocs de silex, charbons...) ou s'il s'agit de structures récentes.

Annoncés par une forte concentration d'éléments dans l'épaisseur du labour, c'est dans les dix premiers centimètres de l'horizon $\mathrm{A}$, non perturbés, que se rencontre le mobilier lithique. Localement, des perturbations font que les vestiges se retrouvent plus en profondeur. Malgré une fouille manuelle et un décapage mécanique intégral, aucun creusement contenant ce mobilier n'a été observé. Si creusements il y a eu, ils étaient superficiels et sont maintenant certainement gommés par la pédogenèse. 
Déjà repérée lors du diagnostic, la nappe de mobilier prend en écharpe l'emprise de la fouille sur une largeur d'environ vingt-sept mètres et une longueur d'au moins quarante-cinq mètres. Les limites septentrionale et méridionale ne sont pas certaines, mais la baisse du nombre de silex enregistré en périphérie suggère qu'elles sont proches. Un petit lot de pièces, découvert au moment du diagnostic dans la tranchée $\mathrm{n}^{\circ} 1$ (fig. 3), à une cinquantaine de mètres du décapage, conduit à envisager l'existence d'autres concentrations sans doute moins étendues et moins riches (Aubry et al., 2004).

Figure 3 : Plan général des faits enregistrés sur le site du Mesnil-Esnard "Les Hautes Haies ». Figure 3 : General plan of the site of Mesnil-Esnard « Les Hautes Haies ».

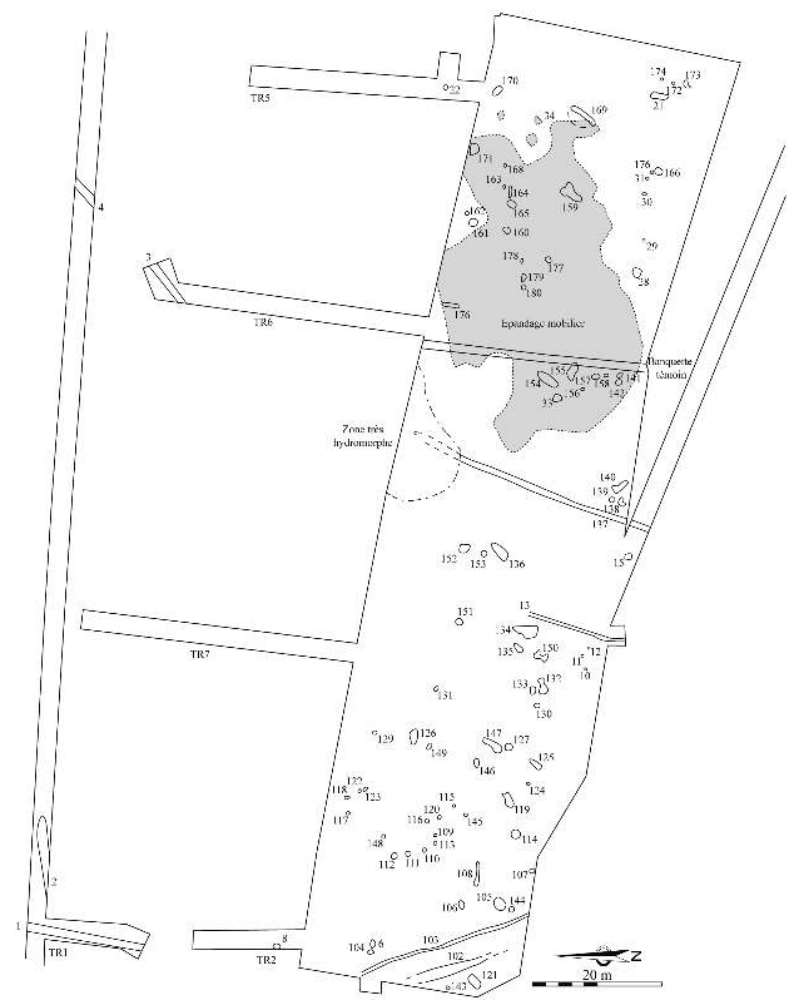

DAO N. Fromont

Ainsi, dans la fenêtre principale, près de mille deux cents mètres carrés sont concernés par ces épandages de silex taillés auxquels s'ajoutent quelques tessons de céramique. Un tiers de la surface a fait l'objet d'un traitement manuel avec enregistrement en trois dimensions des vestiges. Cet exercice, mené sous la forme de sondages, a permis de circonscrire certaines zones pauvres en mobilier qui ont, par la suite, été traitées mécaniquement. Les silex sont frais d'aspect alors que les tessons sont plus abîmés.

\section{Nature et distribution des vestiges de la nappe}

Les vestiges recueillis sont essentiellement liés à des activités de débitage du silex. Il s'agit de plus de mille six cents éléments qui forment un ensemble homogène et cohérent. S'y ajoutent cent quatre-vingts tessons composant un assemblage hétérogène (les éléments modernes ne seront pas décrits ci-dessous).

De prime abord, la répartition du mobilier ne montre aucun phénomène particulier (fig. 4). Les concentrations ne sont jamais très élevées, au mieux une vingtaine de silex 
au mètre carré et en moyenne 3,5 silex par mètre carré (fig.5). La principale concentration a été fouillée lors du diagnostic (Aubry et al., 2004). Les zones de densité moyenne ( 5 à 10 silex au mètre carré) se concentrent le long d'un axe nord-est/sudouest, prenant en écharpe l'emprise de la fouille et recoupant les courbes de niveau de la surface du sol actuel. En revanche, les zones riches (plus de 10 silex) sont plus dispersées. Elles semblent inféodées à des microreliefs et se retrouvent dans la dépression située dans la partie ouest ainsi que dans un petit creux au nord-est de l'emprise. Les quelques tessons protohistoriques sont largement dispersés, avec toutefois un peu plus d'éléments dans la partie est de la nappe (fig. 4). Il n'y a pas de véritable correspondance entre les zones riches en silex et celles riches en céramique. La concentration de tessons notée sur les mètres carrés $\mathrm{R} 44-45$, résulte de la participation d'un seul individu, ce qui relativise l'apparente exclusion entre silex et tessons.

Figure 4 : Mesnil-Esnard «Les Hautes Haies » : nappe de vestiges de la partie est de l'emprise. Figure 4 : Spatial distribution of vestiges in the East part of the excavation.

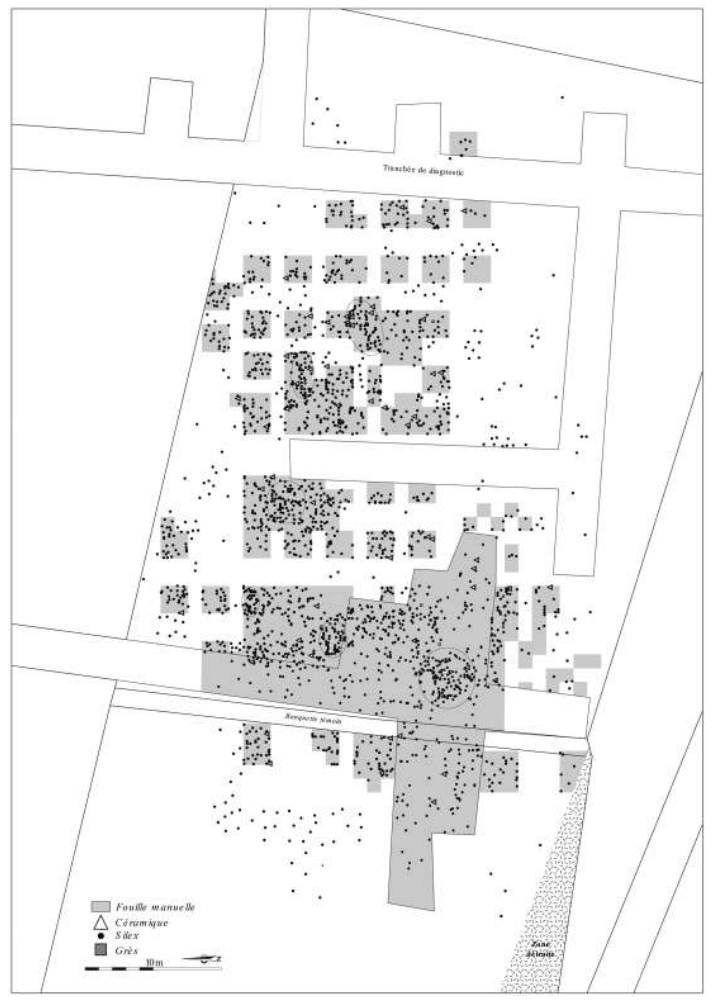

DAO N. Fromont. 
Figure 5 : La nappe de mobilier et la topographie de l'emprise. Figure $5:$ The layer of vestiges and the site topography.

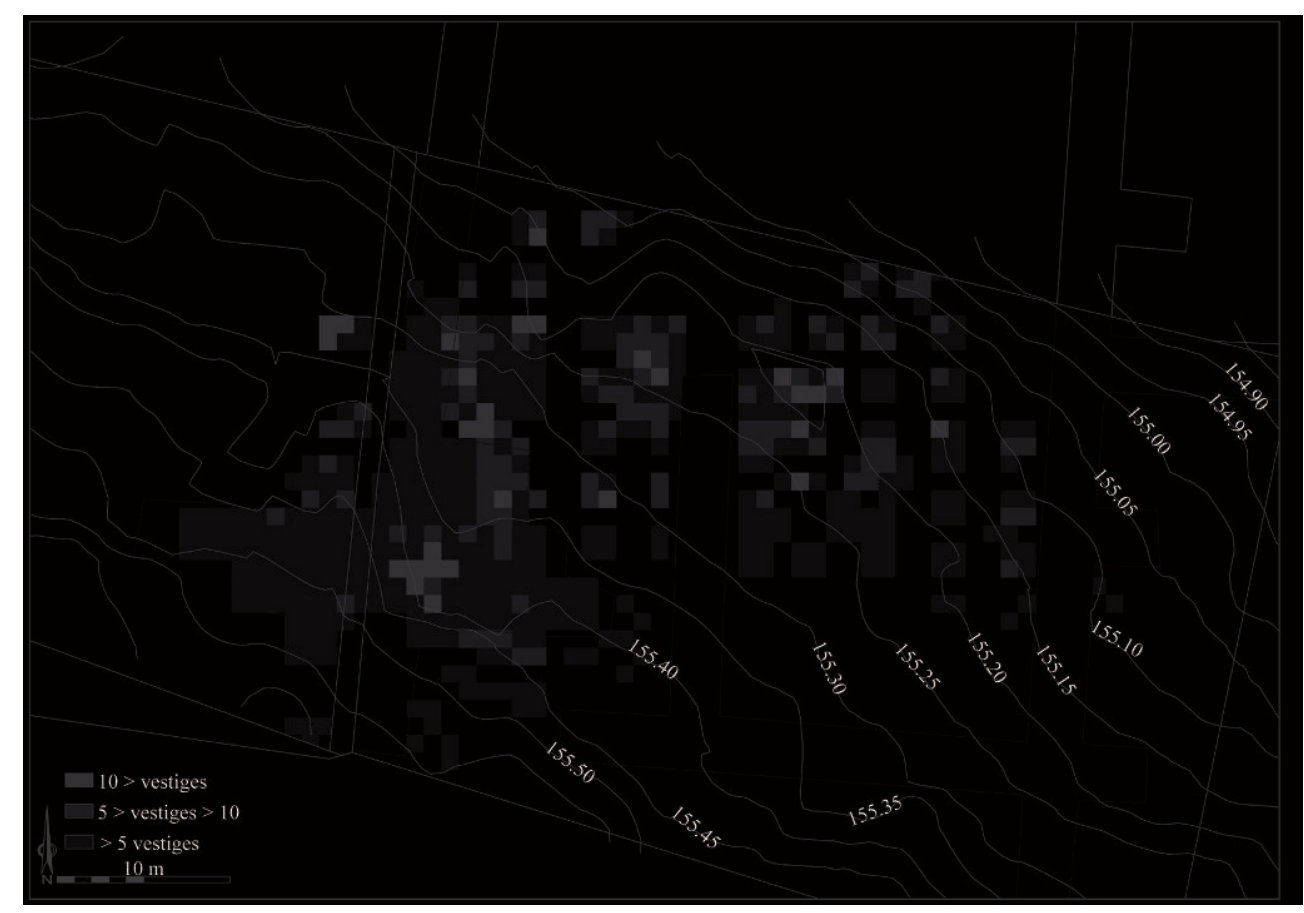

DAO N. Fromont.

Face au peu de résultats escomptés, l'étude de la répartition du mobilier n'a pas été poussée. Une première limite à la portée d'un tel exercice est donnée par les limites de la fenêtre de fouille qui n'a pas permis d'explorer, si ce n'est l'intégralité du site, au moins toute la nappe de vestiges. Ensuite, aucune structure excavée (trous de poteaux, tranchée de fondation, fosses...) ne prouve l'aménagement de l'espace (bâtiment, palissade...). Il ne faut pas pour autant exclure cette possibilité : certaines formes architecturales ne laissent que des traces fugaces. Néanmoins, leur présence (sous la forme de fantômes) n'est aucunement attestée par des effets dans la distribution des vestiges. Face à ces constats, la gestion de l'espace ne pourrait être perçue que par l'analyse de la répartition des vestiges mobiliers et donc des activités qui les ont générés (production/utilisation). Mais ces restes peuvent avoir été remobilisés après leur abandon (nettoyage puis rejet). Autrement dit, nous ne sommes pas assurés qu'ils soient en position primaire. Ils peuvent, en effet, avoir fait l'objet de rejet depuis des aires de débitage et/ou d'utilisation de ces outils. Tenter des remontages serait à même d'apporter des informations relatives à ces questionnements. C'est sans doute une des pistes pour mieux appréhender ces nappes de vestiges.

21 L'étude de la répartition de quelques outils et éléments de la chaîne opératoire de leur production a été réalisée et montre une distribution aléatoire au sein de la nappe. Peutêtre que la reprise des données en multipliant les types d'objets et en croisant leur répartition conduirait à quelques conclusions. Il serait alors essentiel de croiser cette approche avec une étude tracéologique pour caractériser les activités réalisées sur le site. 


\section{L'industrie lithique}

La série lithique du Mesnil-Esnard est constituée du mobilier en silex issu des structures et de la nappe de vestiges (tableau 1). Le mobilier recueilli dans les structures ne permettant pas d'étudier individuellement des lots représentatifs, une approche globale a été privilégiée.

3 Bien que l'on ne soit pas en présence d'un ensemble clos, les caractéristiques typotechnologiques de l'assemblage sont cohérentes et permettent probablement d'exclure un mélange massif d'industries diachroniques. Qu'elles soient issues des structures ou du niveau archéologique, les pièces sont systématiquement d'une grande fraîcheur et la patine est absente.

Tableau 1 : Décompte général de l'assemblage lithique. Table 1 : List of the flint industry.

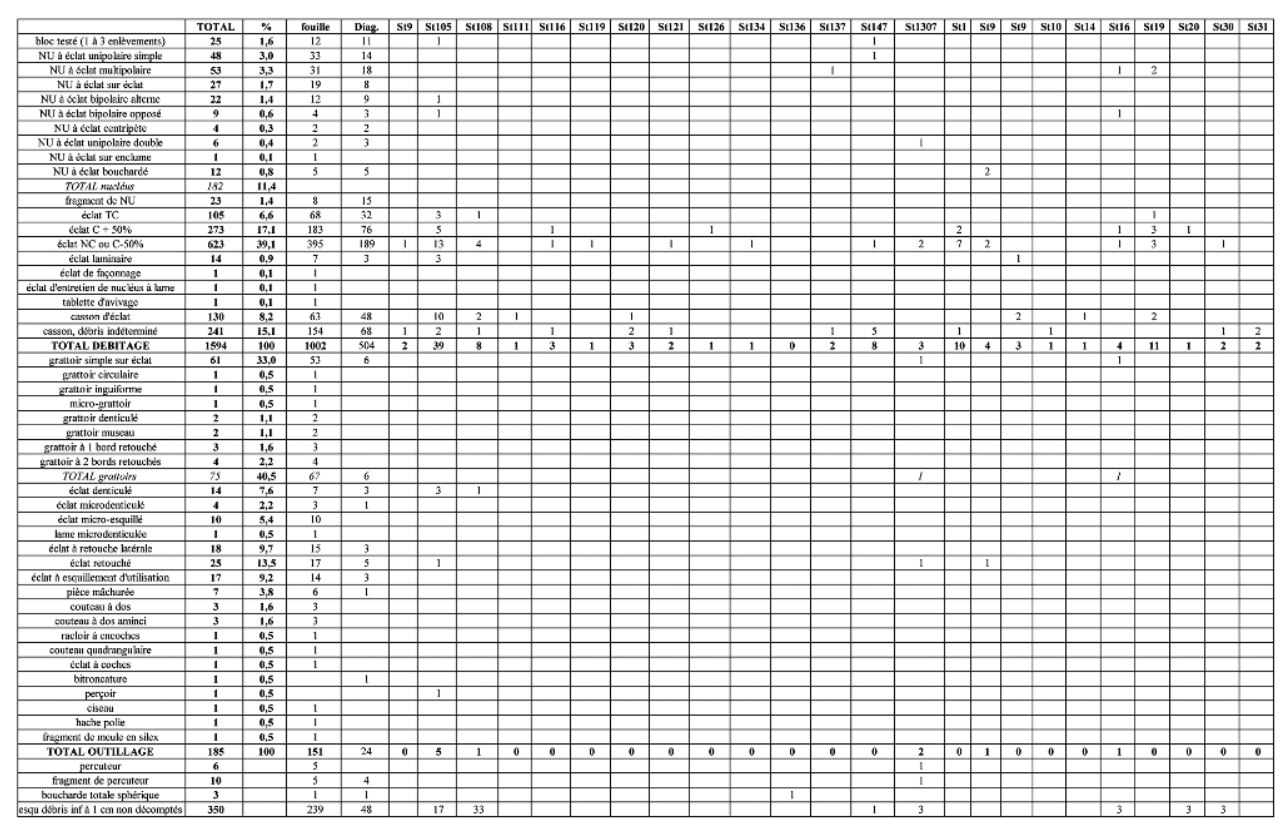

\section{Matière première}

Deux types de silex ont été identifiés (tableau 2). Le plus courant est un silex crétacé dont la couleur varie du gris au noir ( $90 \%$ des nucléus). Les rognons ne sont pas ou peu gélifs et ne posent donc pas de contrainte au débitage, à l'exception de leur morphologie, souvent tourmentée. Ce silex a pu être prélevé en place dans des coteaux crayeux locaux, ou à leurs pied dans des talus d'éboulis. Trois qualités différentes au sein de ce matériau ont été distinguées, bien que celles-ci ne semblent pas avoir eu d'influence sur la qualité du débitage : de couleur gris clair, avec un grain fin, mat et opaque ( $43 \%$ des nucléus); de couleur gris foncé, avec un grain fin, mat et opaque $(36,2 \%$ des nucléus) ; de couleur noire, avec un grain très lisse et translucide (matériau d'excellente qualité clastique, représentant 10,6 \% des nucléus). 
Tableau 2 : Matériaux siliceux débités (NU : nucléus).

Table 2 : Siliceous raw materials (NU: core).

\begin{tabular}{|c|c|c|c|c|}
\cline { 2 - 5 } \multicolumn{1}{c|}{} & \multicolumn{2}{c|}{ crétacé sub-local de bonne qualité } & silex local chailleux \\
\cline { 2 - 5 } \multicolumn{1}{c|}{} & gris clair & gris foncé & noir & beige \\
\hline Bloc testé & 12 & 10 & & 3 \\
\hline NU à éclat unipolaire simple & 13 & 19 & 8 & 8 \\
\hline NU à éclat multipolaire & 26 & 18 & 2 & 7 \\
\hline NU à éclat sur éclat & 13 & 6 & 7 & 1 \\
\hline NU à éclat bipolaire alterne & 6 & 7 & 1 & \\
\hline NU à éclat croisé & 3 & 5 & & \\
\hline NU à éclat bipolaire opposé & 5 & 2 & 2 & \\
\hline NU à éclat centripète & 3 & 1 & & \\
\hline NU à éclat unipolaire double & 3 & 1 & 2 & \\
\hline NU à éclat sur enclume & & 1 & & 21 \\
\hline NU à éclat bouchardé & 5 & 5 & & 10,1 \\
\hline TOTAL & 89 & 75 & 22 & \\
\hline$\%$ & 43,0 & 36,2 & 10,6 & \\
\hline
\end{tabular}

Enfin, un silex beige, généralement chailleux et gélif, a aussi été ponctuellement utilisé (10 \% des nucléus). Ce matériau issu d'argiles à silex locales a certainement été exploité en raison de sa disponibilité dans l'environnement immédiat du site.

\section{Le débitage}

Le débitage est illustré par 1412 produits divers, hors esquilles (tableau 1), auxquels s'ajoutent 182 nucléus à éclats, ainsi que 25 blocs testés (tableau 2). Les nucléus à lame ou à lamelle sont totalement absents.

\section{Les différents types de nucléus à éclat}

Les nucléus unipolaires simples (48 pièces) exploitent les convexités naturelles du rognon, avec une table généralement peu étendue, parfois légèrement tournante. Le plan de frappe est presque systématiquement lisse, obtenu à partir d'un seul éclat d'initialisation. Le débitage pourrait vite être confronté à une angulation insuffisante. Pourtant, les rebroussés sont peu nombreux sur ce type de nucléus. Les tailleurs ont volontairement interrompu le débitage avant d'être confrontés à une interdiction technique. Il en résulte une exploitation des volumes très largement médiocre, avec un faible nombre de produits et des supports souvent partiellement corticaux.

Les nucléus sur éclat (27 pièces) sont toujours unipolaires. Leur présence s'explique par le volume tourmenté des blocs, qui génère des éclats d'initialisation volumineux dont les convexités sont faciles à exploiter à partir de leur face d'éclatement.

L'exploitation unipolaire domine dans la série, d'autant plus que les nucléus "sur éclat » sont quasi systématiquement de ce type. En incluant ces derniers, les nucléus unipolaires simples comptent soixante-quinze individus, soit $41 \%$ des nucléus.

Les nucléus multipolaires (53 pièces) sont, avec la méthode unipolaire, la catégorie la mieux représentée. Les plans de frappe sont multipliés en exploitant les surfaces dégagées par le débitage. Au terme de cette exploitation, quand les angulations 
approchent de quatre-vingt-dix degrés, les nucléus adoptent une morphologie globuleuse, mais cet état d'exhaustion n'est pas systématiquement rencontré.

31 Le reste du corpus est composé de méthodes diverses, tributaires d'un opportunisme dans la gestion des volumes. Ainsi, on trouve des nucléus bipolaires alternes (22 pièces), qui s'apparentent à deux sessions unipolaires consécutives, le plan de frappe de la dernière exploitant la table de la précédente. Les autres méthodes sont faiblement représentées : nucléus bipolaires opposés (9 pièces), qui correspondent à deux sessions unipolaires successives; nucléus centripètes (4 pièces), évolution tournante de la méthode bipolaire alterne; nucléus unipolaires doubles (6 pièces), possédant deux plans de frappe distincts à la faveur des volumes du bloc. Enfin, il faut signaler un petit rognon ovoïde fracturé sur enclume, selon la technique habituellement dévolue aux industries exploitant les galets de silex côtier.

Douze nucléus multipolaires présentent des surfaces bouchardées variant entre 1 et 5 centimètres carrés. La morphologie, le poids (entre 52 et 304 grammes) et le module de ces objets les rendent tout à fait utilisables comme percuteurs dans le cadre du débitage du silex.

\section{Les produits débités}

Le débitage est illustré par 1015 produits, auxquels s'ajoutent 130 cassons d'éclats et 241 cassons et débris indéterminables.

Les produits corticaux (378 pièces) attestent de l'initialisation des blocs sur le site. Des éclats totalement corticaux sont présents (105 pièces). Les éclats dont plus de la moitié de la surface est corticale sont nombreux (273 pièces). Ceci s'explique par la petitesse des rognons mis en œuvre et par le faible développement de la chaîne opératoire. Ces supports corticaux sont exploités dans le cadre de l'outillage.

Les produits les plus fréquents sont les éclats non ou peu corticaux (623 pièces). Leur longueur moyenne oscille entre 4 et 5 centimètres, pour une largeur souvent comparable. Les talons sont généralement larges et lisses (tableau 3), avec des traces de préparation au débitage quasi inexistantes: seule une minorité d'éclats a subi une réduction de la corniche. L'utilisation systématique du percuteur dur est à l'origine d'une proportion non négligeable de cassons d'éclats ( $9 \%$ des produits débités).

Tableau 3 : Types de talons des produits débités (éclats corticaux et non corticaux). Table 3 : Types of heels of the debitage products (Cortical and non corticals).

\begin{tabular}{|c|c|c|}
\cline { 2 - 3 } \multicolumn{1}{c|}{} & TOTAL & \% \\
\hline cortical & 124 & 12,2 \\
\hline lisse & 699 & 68,9 \\
\hline dièdre & 76 & 7,5 \\
\hline facetté & 28 & 2,8 \\
\hline punctiforme & 88 & 8,7 \\
\hline
\end{tabular}


36 Les produits laminaires sont anecdotiques (14 pièces). Obtenus sans préparation véritable (tout au plus une légère réduction de la corniche), ces produits intègrent la chaîne opératoire de production d'éclats. Si elle n'est pas involontaire, leur production est largement opportuniste. On ne peut pas considérer qu'il existe dans la série une véritable production de supports allongés. La présence dans l'outillage d'une lame véritable est une exception. Obtenue à partir d'une préparation volumétrique et d'un débitage parfaitement maîtrisé, cette lame est sans doute arrivée sur le site sous la forme d'un produit fini.

\section{Bilan du débitage}

37 Les rognons de silex exploités sont de volumes modestes, avec une rentabilité souvent médiocre. La présence de blocs simplement testés (ou très peu exploités) plaide en faveur d'une relative proximité et abondance du silex crétacé utilisé.

Le débitage consiste en la production sur place d'éclats de module modeste, à partir d'une chaîne opératoire très simplifiée, unipolaire et exploitant les convexités naturelles. L'examen des talons des produits débités confirme cette simplicité opératoire : les talons lisses dominent (68,9\%).

Les quelques produits laminaires obtenus et parfois utilisés dans l'outillage ne relèvent pas d'une chaîne opératoire spécifique.

En finalité, aucune véritable prédétermination du débitage n'est perceptible, mais les techniques mises en œuvre sont en adéquation avec la matière première et le type de produits recherchés. Accessible à tous, ce débitage de faible investissement technique contraste avec l'unique lame de la série qui témoigne d'un savoir-faire spécifique et sans doute moins répandu au sein d'une communauté.

\section{L'outillage}

41 L'outillage est représenté par 185 objets, associant des outils retouchés ou façonnés et des outils a posteriori porteurs d'enlèvements ou d'esquillements non intentionnels mais trahissant leur utilisation.

\section{Les outils retouchés ou façonnés}

Les grattoirs (61 pièces) dominent largement l'assemblage. La diversité typologique est importante au sein de cette catégorie et plusieurs sous-types ont été différenciés (fig. 6 et 7). Mais cette variabilité peut être tributaire de fonctions distinctes, d'un opportunisme dans le choix du support ou d'un état d'exhaustion. Il est impossible de trancher en l'absence d'une étude tracéologique. 
Figure 6 : Mesnil-Esnard "Les Hautes Haies », industrie en silex : grattoirs. Figure 6 : Mesnil-Esnard "Les Hautes Haies", flint industry: scrapers.
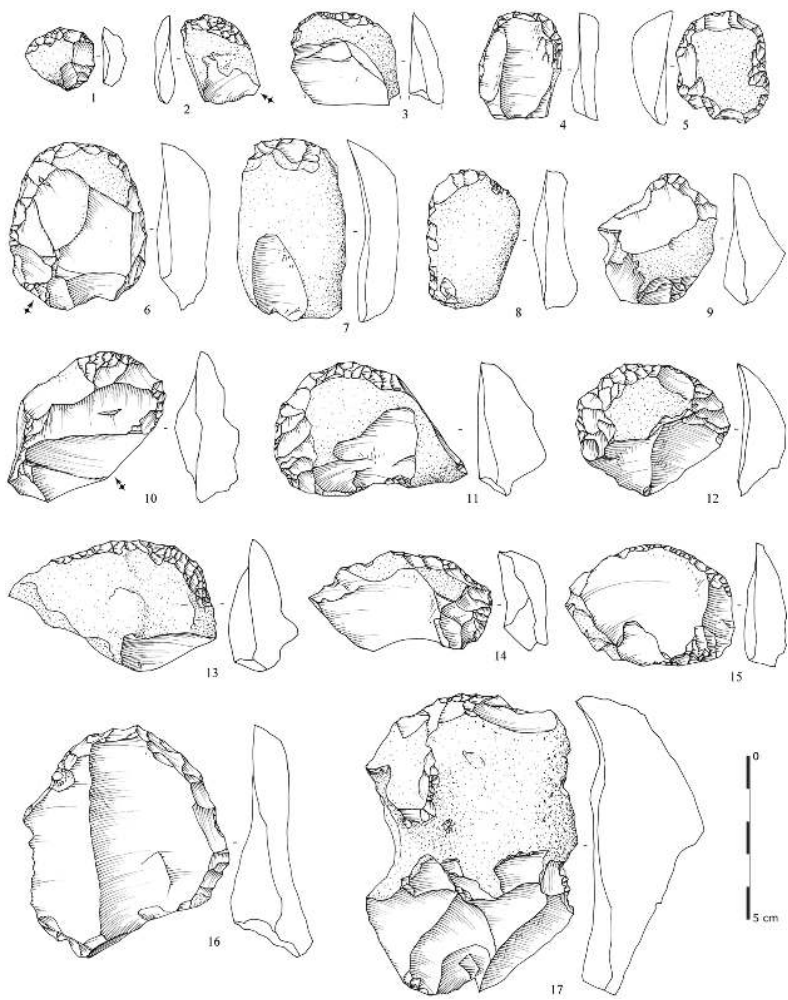

Dessin, DAO L. Juhel. 
Figure 7 : Mesnil-Esnard "Les Hautes Haies », industrie en silex : grattoirs. Figure 7 : Mesnil-Esnard "Les Hautes Haies", flint industry: scrapers.
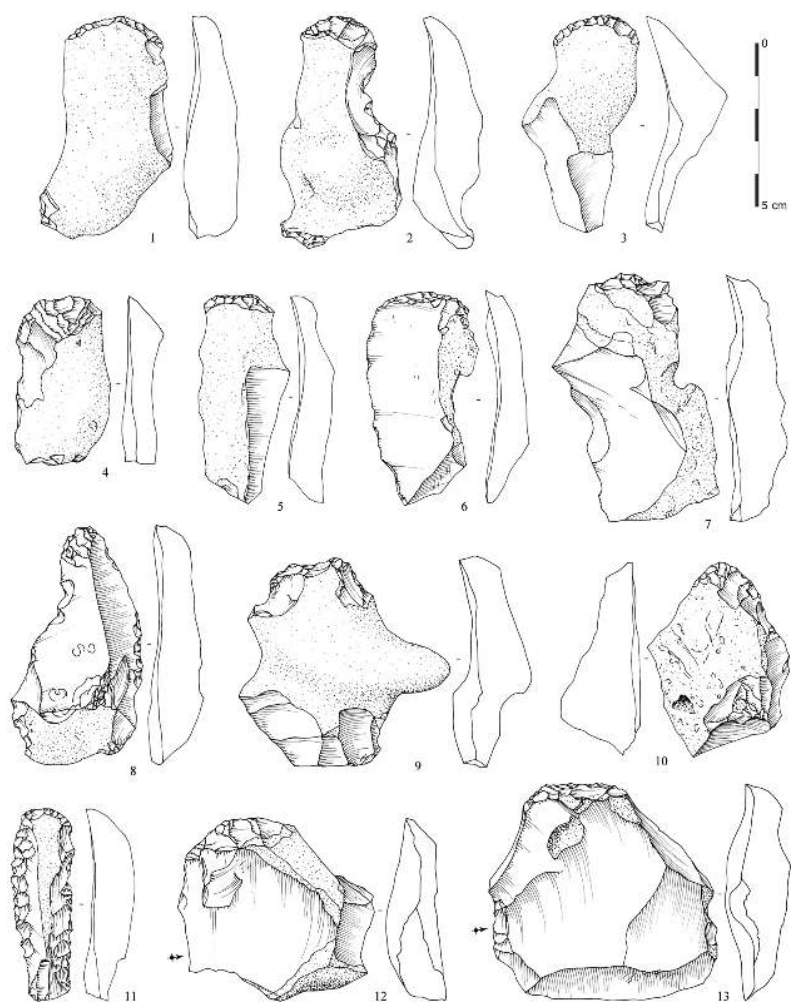

Dessin, DAO L. Juhel.

Les retouches sont semi-abruptes à abruptes, quelquefois obliques. Les supports sont de dimensions et de morphologies variées. On peut signaler quelques pièces aménagées sur des supports allongés et corticaux, peut-être à mettre en relation avec une fonction ou une utilisation spécifique (fig. $7, \mathrm{n}^{\circ} 1$ à 8). Un « micro-grattoir » se caractérise, quant à lui, par ses petites dimensions et sa faible épaisseur (fig. $6, n^{\circ} 2$ ); il peut être rapproché d'un autre grattoir rapportable au type unguiforme (fig. 6, $n^{\circ} 1$ ). Enfin, une autre pièce isolée se distingue par une bitroncature de son support, façonnant un outil allongé, peut-être en vue d'une utilisation emmanché (fig. $\left.7, \mathrm{n}^{\circ} 11\right)$. Son front très court est affecté par un lustré d'utilisation sur la face inférieure.

Les éclats à retouches latérales (18 pièces) sont des pièces à un ou deux bords retouchés, traditionnellement rapportées au type "racloir ». Mais cette catégorie rassemble des pièces aux usages sans doute différents. La distinction entre racloir et couteau à dos pose parfois la difficulté de la reconnaissance du bord actif à l'œil nu, sauf quand le bord retouché est opposé à un dos cortical (fig. $8, n^{\circ} 2$ et 3 ). 
Figure 8 : Mesnil-Esnard "Les Hautes Haies", industrie en silex : éclats à retouches latérales. Figure 8 : Mesnil-Esnard "Les Hautes Haies", flint industry: flakes with lateral retouches.
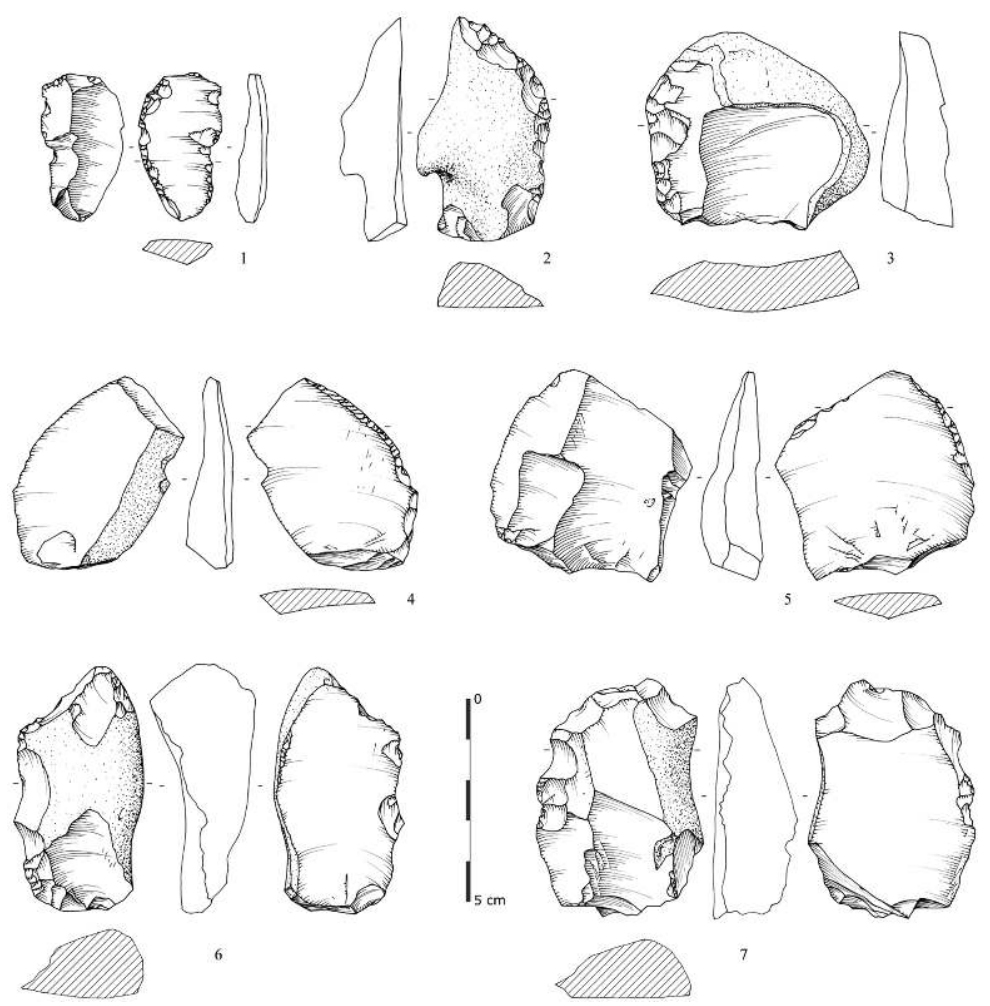

Dessin, DAO L. Juhel.

Quatre pièces portent des retouches très obliques, fines et légèrement écailleuses. Le bord est très régulier, probablement obtenu au percuteur tendre (fig. $8, \mathrm{n}^{\circ} 4$ et 5 ).

Deux pièces portent des enlèvements écailleux sur leur face inférieure, à partir d'un bord retouché (fig. $8, \mathrm{n}^{\text {os }} 6$ et 7 ). Une d'entre elles comporte un écrasement par esquillement du côté opposé au tranchant retouché (fig. 8, no 6). À partir de ces stigmates, une utilisation comme coin à fendre peut être envisagée.

Les denticulés (14 pièces) exploitent des éclats irréguliers et relativement épais (jusqu'à $38 \mathrm{~mm}$ ). Les dents sont obtenues par des coches souvent assez profondes et régulièrement espacées dans certains cas (fig. $9, \mathrm{n}^{\circ} 2$ ). Une pièce se distingue par ses grandes dimensions et des denticulations régulières présentes sur les deux tiers de son pourtour (fig. $9, \mathrm{n}^{\circ} 3$ ). 
Figure 9 : Mesnil-Esnard "Les Hautes Haies », industrie en silex : denticulés. Figure 9 : Mesnil-Esnard "Les Hautes Haies", flint industry: denticulates.
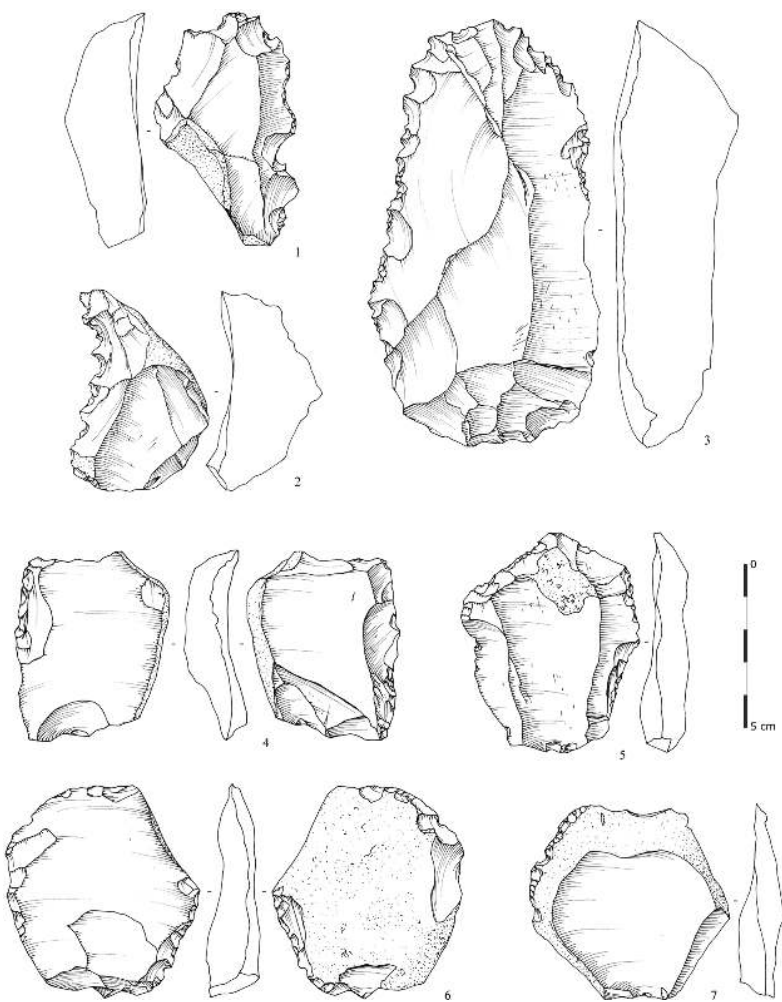

Dessin, DAO L. Juhel.

Les couteaux à dos (3 pièces) sont réalisés sur des éclats allongés ou laminaires (fig. 10, $\mathrm{n}^{\circ} 1$ à 3$)$. Opposé à un tranchant brut, un bord est aménagé par une retouche semiabrupte qui définit un dos courbe. Deux pièces voient leur tranchant affecté de fins esquillements d'utilisation. Dans un cas, ces stigmates s'accompagnent d'un lustré marginal développé sur les deux faces du tranchant (fig. 10, $\mathrm{n}^{\circ} 3$ ). 
Figure 10 : Mesnil-Esnard « Les Hautes Haies », industrie en silex. Figure 10 : Mesnil-Esnard "Les Hautes Haies", flint industry.
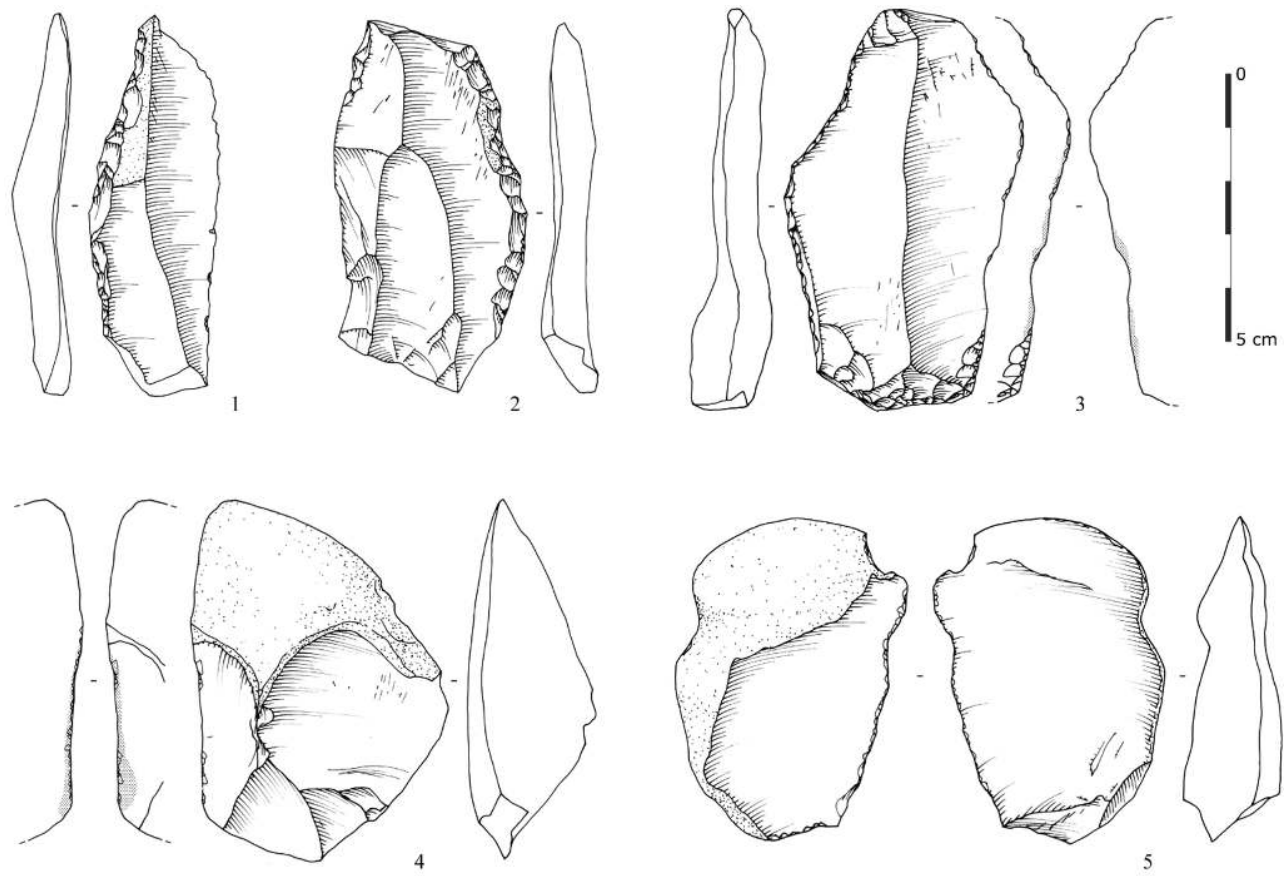

1 à 3 : couteaux à dos retouché ; 4 et 5 : éclats à dos naturel et tranchant micro-esquillé 1 to 3: retouched back knives; 4 and 5: flakes with natural back and sharp scars.

Dessin, DAO L. Juhel.

La hache polie est représentée par un fragment distal en silex gris (fig. $11, \mathrm{n}^{\circ} 1$ ). La partie observable du corps de la hache est partiellement polie, conservant de larges surfaces brutes de façonnage. Les chants latéraux ont été aplanis par le polissage. Le tranchant arrondi et légèrement vrillé est soigneusement affuté et lustré. Les bords très parallèles $\mathrm{du}$ fragment permettent d'exclure une silhouette évasée; celle-ci devait être relativement rectiligne. Par comparaison de son rapport longueur/largeur avec des exemplaires entiers, on peut envisager une longueur d'une vingtaine de centimètres.

Figure 11 : Mesnil-Esnard « Les Hautes Haies », industrie en silex. 


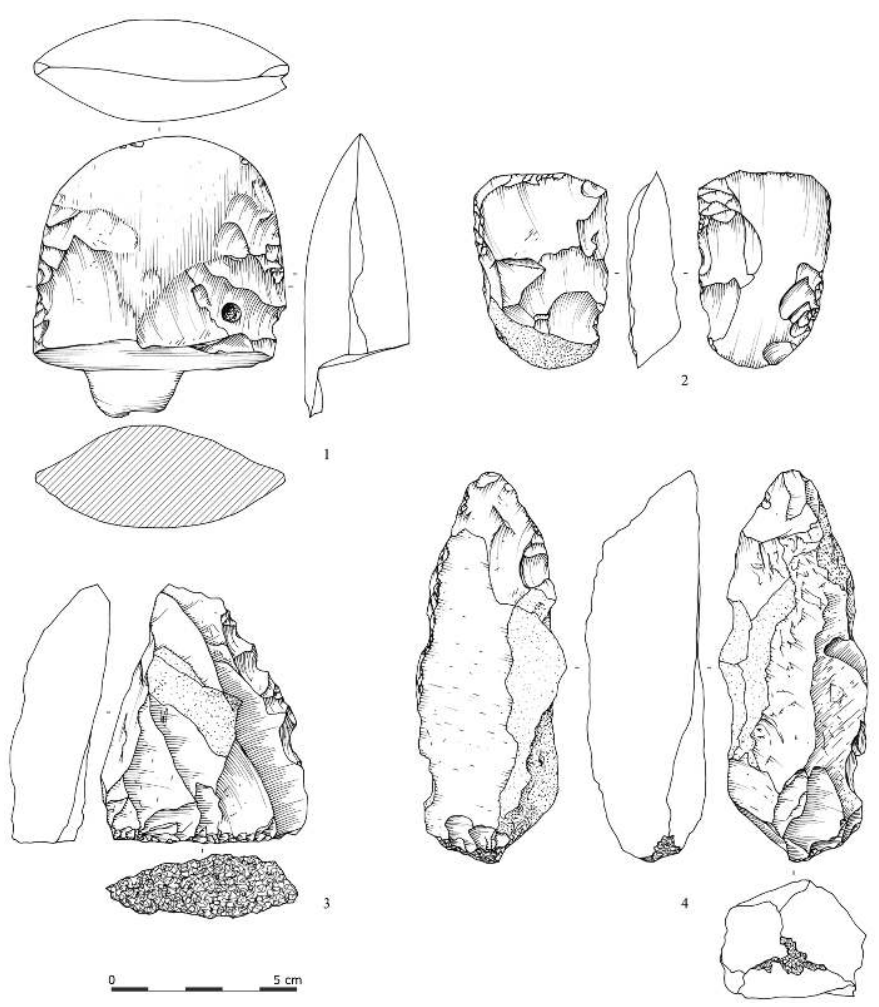

Lame de hache polie (1), bi-troncature (2), éclat de meule (3) et ciseau (4). Polished axe (1), "bitroncature" (3), flake of millstone, pick (4)

Dessin, DAO L. Juhel.

La bitroncature est assimilable à un petit tranchet obtenu à partir d'un éclat (fig. 11, $\mathrm{n}^{\circ} 2$ ). Le tranchant distal est brut et rectiligne. Les retouches latérales façonnant la silhouette sont partiellement bifaciales (retouches semi abruptes directes et inverses envahissantes).

Le ciseau est façonné à partir d'un fragment long de silex (fig. 11, $\mathrm{n}^{\circ} 4$ ). Les surfaces corticales sont largement préservées, un seul côté étant travaillé par une retouche abrupte définissant une section polygonale. L'extrémité distale a une silhouette qui évoque un pic, mais elle est issue d'un raffûtage raté, suite à deux enlèvements type coup de tranchet mal maîtrisés. L'extrémité opposée est esquillée et bouchardée par une percussion répétée à la pierre. Il pourrait s'agir d'une préparation (?) en vue d'encaisser convenablement les impacts d'un maillet, dans le cadre d'une utilisation du ciseau en préhension directe et percussion posée.

Le perçoir est réalisé à partir d'un éclat robuste. C'est un perçoir double, avec les deux pointes opposées dans l'axe de la largeur du support. Les pointes sont dégagées par une retouche écailleuse semi-abrupte qui leur donne une section triédrique.

L'éclat à coche affecté de deux encoches profondes, obtenues par une retouche régulière directe dans un cas, inverse dans l'autre.

Le fragment de meule en silex est un éclat provenant d'une pièce en silex gris foncé, très légèrement bleuté (fig. $11, \mathrm{n}^{\circ} 3$ ). Son grain, un peu moins lisse, diffère des autres matières premières crétacées débitées sur le site. Une origine cénomanienne dans des formations en falaise côtière du secteur du Havre est probable, bien que l'on ne puisse 
exclure les formations rouennaises du cénomanien (Watté 2004). Un lambeau de surface piquetée et émoussée constitue le talon de cet éclat. Ainsi, il apparaît que l'objet de mouture initial a été débité au moins partiellement à partir de sa surface de broyage. Le bord de cet éclat a ensuite été transformé en denticulé.

Ce type d'objet, toujours dans le même silex cénomanien, est régulièrement rencontré en Normandie, dans des contextes chronologiques pouvant s'étaler entre le Néolithique final et La Tène ancienne. À titre d'exemple, l'ensemble 17 de Saint-Vigor-d'Ymonville, rapportée à la deuxième moitié du III ${ }^{e}$ millénaire, a livré dix-neuf fragments de meule en silex similaire (Marcigny et al., 2002).

\section{Les pièces rapportables au couteau à moissonner}

Le racloir à encoche a été mis en forme à partir d'un éclat épais en silex chailleux local (fig. $12, \mathrm{n}^{\circ} 1$ ). Les encoches sont aménagées sur le talon et l'extrémité distale du support par une retouche directe. La silhouette de l'outil est légèrement trapézoïdale. Le tranchant le plus long (bord actif?) porte une retouche directe irrégulière et discontinue; le tranchant opposé comporte quant à lui une retouche inverse sur l'ensemble de sa longueur.

Figure 12 : Mesnil-Esnard " Les Hautes Haies », industrie en silex.

Figure 12 : Mesnil-Esnard "Les Hautes Haies", flint industry.

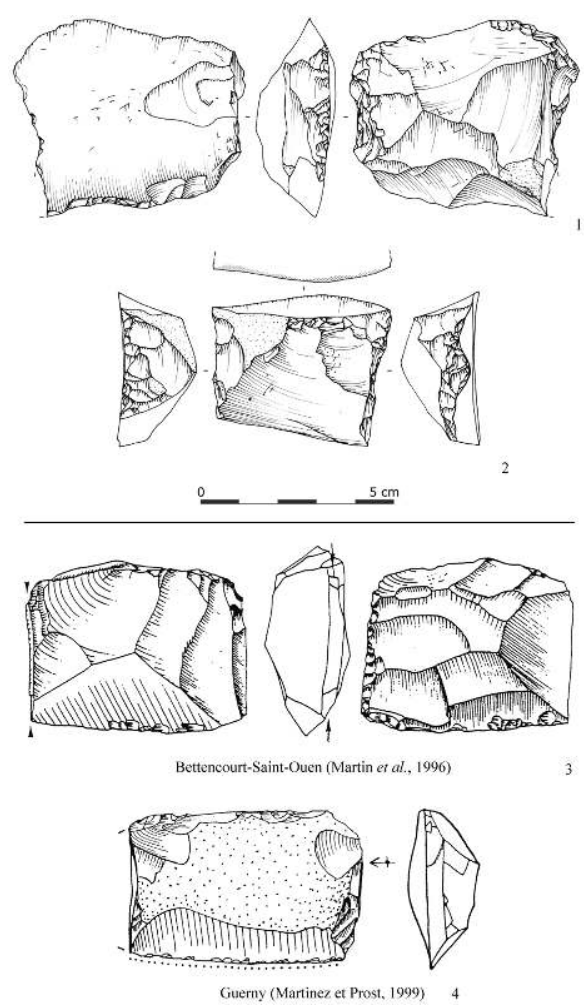

1 : racloir à encoche ; 2 : couteau quadrangulaire ; 3 et 4 : exemples de pièces d'autres sites assimilables aux couteaux quadrangulaires. 7: scraper with notch; 2 to 4: quadrangular knife. DAO L. Juhel sauf $n^{\circ} 3$ et 4 : D. Prost. 
57 objet de silhouette quadrangulaire légèrement trapézoïdale (fig. 12, $n^{\circ} 2$ ), à tranchant non retouché. Les deux côtés sont façonnés par une retouche abrupte à semi-abrupte, directe dans un cas, directe à partir de deux enlèvements inverses dans l'autre. Le bord opposé au tranchant est laissé brut (angulation proche de 90 degrés). Le tranchant porte un léger lustré sur la face inférieure.

D. Prost a été le premier à décrire dans l'outillage du site de Bettencourt-Saint-Ouen dans la Somme (Martin et al., 1996), en contexte du Néolithique final, deux pièces de forme rectangulaire, avec un tranchant large, épais et rectiligne (fig. $12, \mathrm{n}^{\circ} 3$ ). Ces pièces sont alors comparées aux racloirs à encoches. Le même auteur décrit également, pour le site de Guerny, dans l'Eure (Martinez et Prost, 1999), un couteau de forme rectangulaire très régulière (fig. $12, \mathrm{n}^{\circ} 4$ ), dont le dos est retouché, avec un bord tranchant émoussé et lustré. dessus, bien que de dimensions légèrement inférieures. Une utilisation en couteau à moissonner est tout à fait envisageable. Le tranchant de ces objets n'étant pas systématiquement retouché, nous proposons ici d'introduire le terme de «couteau quadrangulaire $»$.

Les couteaux à dos aminci (3 pièces) sont différenciés des classiques couteaux à dos en raison d'une morphologie distincte et d'une technique de façonnage singulière. Les dos sont légèrement courbes, deux étant aménagés par retouche directe et inverse (fig. 13, $\mathrm{n}^{\circ} 2$ et 3 ) et seulement inverse dans un cas (fig. 13, $\mathrm{n}^{\circ} 1$ ). Ce type de retouche évoque un aménagement lié à un emmanchement: les retouches inverses permettent d'amincir l'éclat et de corriger son cintrage. Elles pourraient ainsi faciliter l'adaptation et l'insertion dans un manche rainuré (fig. $13, \mathrm{n}^{\circ} 4$ ) comme, par exemple, ceux observés dans la culture Horgen (Honegger, 2001). 
Figure 13 : Mesnil-Esnard « Les Hautes Haies », industrie en silex. Figure 13 : Mesnil-Esnard "Les Hautes Haies", flint industry.
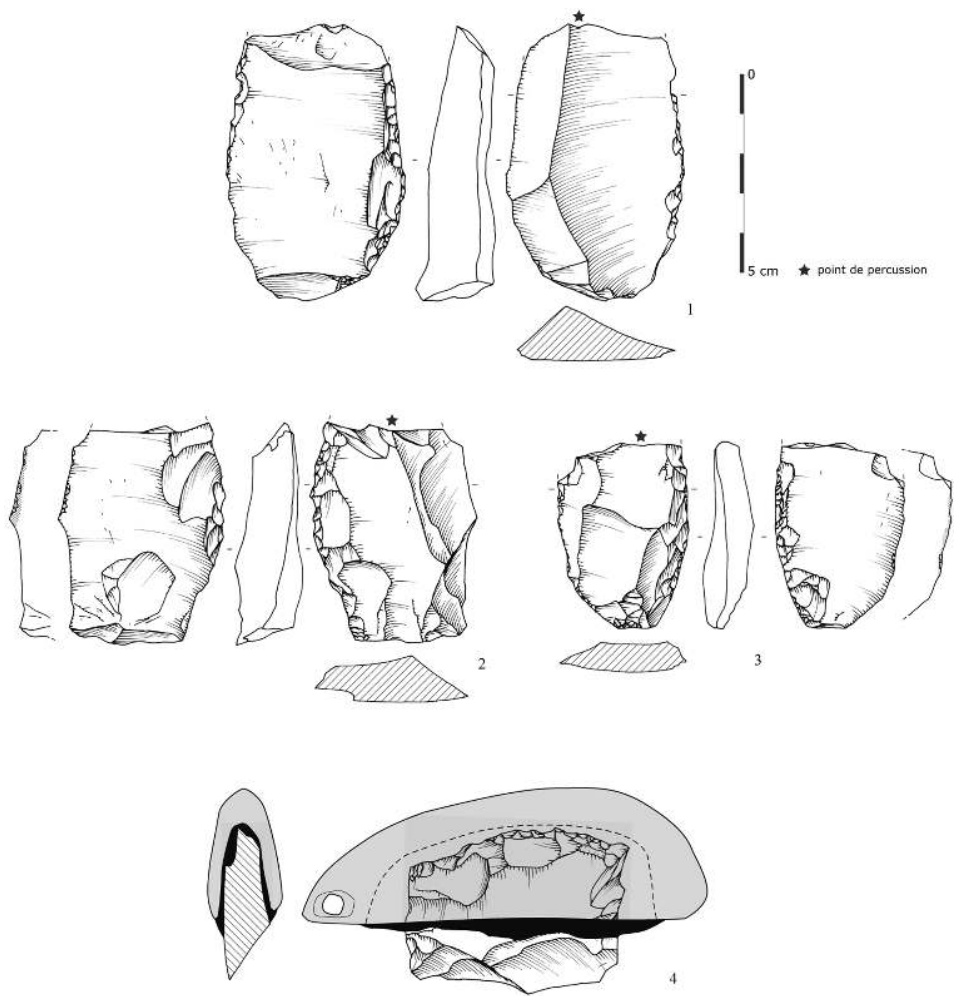

1 à 3 : couteaux à dos aminci ; 4 : hypothèse d'emmanchement.

1 to 3: knives with thinned back; 4 : handle hypothesis.

Dessin, DAO L. Juhel.

61 La seconde particularité de ces pièces à dos est la technique de troncature par "fracturation » de leur extrémité distale. Pour les trois pièces, la cassure est réalisée par un impact sur la face supérieure de l'éclat (peut-être en plaçant la pièce en porte à faux), postérieurement au façonnage du dos. Les extrémités proximales conservent le talon de l'éclat, sans autre aménagement. La troncature permet de contrôler le module de ces pièces et d'obtenir une silhouette rectangulaire.

Sur deux pièces, des luisants marginaux sont observables sur le tranchant, en plus de micro-esquillements d'utilisation.

À Bettencourt-Saint-Ouen, les auteurs mentionnent plusieurs pièces à dos sur éclats larges, dont les dimensions et les formes se rapprochent de celles des racloirs à encoches (Martin et al., 1996).

En conclusion, il semble intéressant d'insister sur l'association dans l'outillage du Mesnil-Esnard d'un racloir à encoche, d'un «couteau quadrangulaire " et de trois "couteaux à dos aminci». D'un point de vue typologique (avec les limites que cela comporte), ces objets semblent rapportables aux couteaux à moissonner connus sur les sites de la fin du Néolithique. Pour une fonction similaire, les divergences morphologiques pourraient s'expliquer par différents modes de préhension ou d'emmanchement. 


\section{Les microdenticulés et pièces assimilables}

Les microdenticulés (4 pièces) sont réalisés sur des supports de morphologies variées (fig. 14, $\mathrm{n}^{\text {os }} 1$ à 4 ). Les retouches sont directes dans deux cas, inverses pour les deux autres. La fracture brachiale est rarement développée; souvent, seul l'ombilic est présent, accompagné d'un enlèvement de très faible dimension. L'espacement des dents sur le tranchant est très variable. Des lustrés marginaux recouvrent les tranchants dans deux cas. Une pièce porte une retouche inverse, écailleuse du bord, opposée au tranchant (fig. 14, $\mathrm{n}^{\circ} 3$ ). Cette retouche redresse le profil du bord concerné et peut être comparée aux aménagements des couteaux à dos aminci.

La lame microdenticulée est le seul véritable produit laminaire de la série (fig. $14, \mathrm{n}^{\circ}{ }^{5}$ ). La quasi-totalité de son pourtour est affecté de microdenticulations directes ou inverses. Les coches sont très petites et particulièrement serrées les unes contre les autres sur une portion distale du tranchant. On observe également quelques esquillements d'utilisation, ainsi qu'un luisant très marginal, superposés à certaines retouches.

Figure 14 : Mesnil-Esnard "Les Hautes Haies », industrie en silex : microdenticulés. Figure 14 : Mesnil-Esnard "Les Hautes Haies", flint industry: microdenticulates.
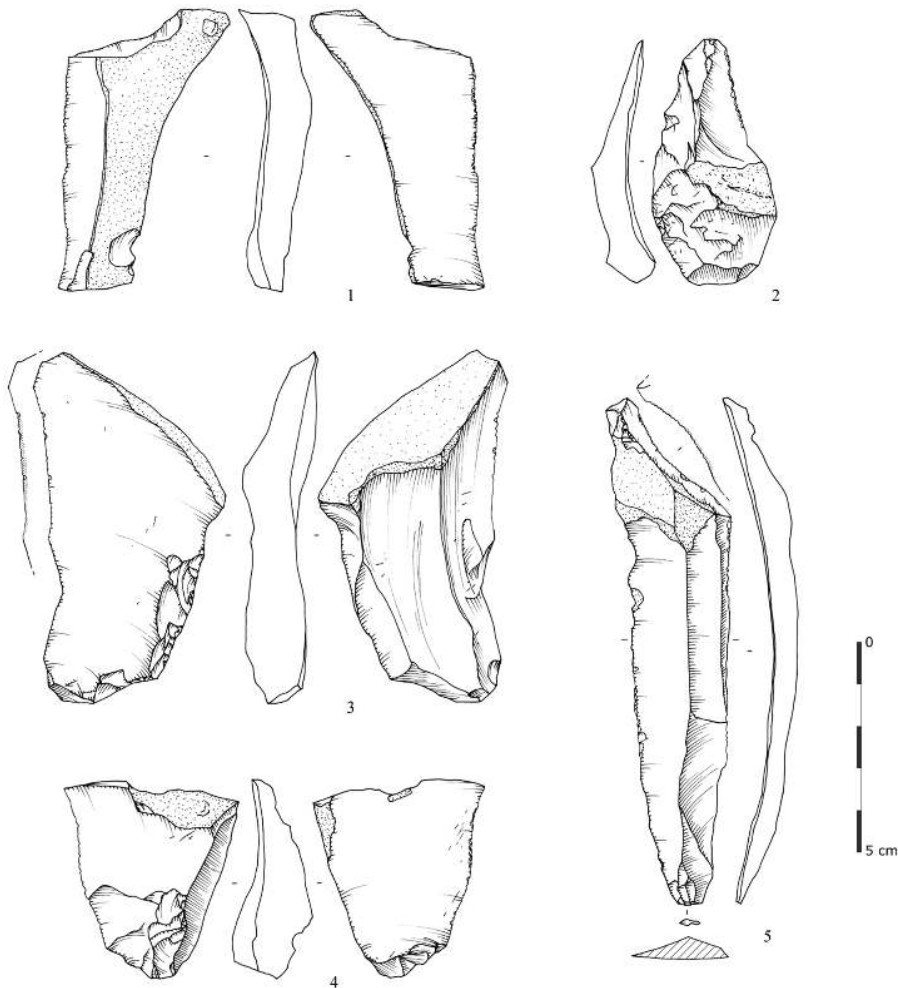

Dessin, DAO L. Juhel.

Les éclats micro-esquillés (10 pièces) correspondent à des supports dont le tranchant comporte de très fins esquillements d'utilisation, directs et/ou inverses (fig. 10, $\mathrm{n}^{\circ} 4$ et 5). Sur plusieurs pièces, quelques enlèvements s'apparentent à des coches de microdenticulé sans, toutefois, aucune régularité. Les tranchants sont souvent lustrés et parfois même émoussés. Deux couteaux à dos sont affectés de stigmates similaires (fig. $10, n^{\circ} 2$ et 3 ). 
$68 \mathrm{Au}$ stade de l'observation macroscopique, certaines pièces évoquent des microdenticulés ayant subi une usure poussée de leur tranchant initial. D'autres pièces sont exemptes de microdenticulations, mais leur aspect évoque une action transversale sur végétaux non ligneux (observations macroscopiques et communication orale J.P. Caspar), identique à celle classiquement observée à fort grossissement sur les microdenticulés typiques.

69 Finalement, il est délicat de quantifier la part de microdenticulés au sein de la série du Mesnil-Esnard. Les pièces véritablement typiques sont en effet mal représentées, mais les éclats micro-esquillés semblent pouvoir être rapprochés de cette catégorie. Le pourcentage faible des microdenticulés «véritables » $(2,7 \%)$ passe à $8,1 \%$ si l'on y inclut les éclats micro-esquillés.

\section{Les outils a posteriori}

70 Les éclats à esquillement d'utilisation (17 pièces) sont de morphologies très diverses, avec une majorité de supports assez épais. L'ampleur des esquillements est variable. La délinéation du bord portant les traces est le plus souvent accidentée, avec des enlèvements écailleux isolés ou se chevauchant.

71 Les éclats retouchés (25 pièces) n'ont pas pu être attribués à des types précis d'outils. Les retouches pourraient participer à l'aménagement de fronts actifs ou de parties emmanchées.

Les pièces mâchurées (7 pièces) sont des éclats de module varié, toujours épais. Un fragment de nucléus a aussi été utilisé. Les surfaces mâchurées correspondent à des portions d'arêtes plus ou moins étendues, qui ont subi des percussions répétées, dont la résultante est un esquillement important, évoluant parfois jusqu'à la surface bouchardée. La fonction de ces pièces reste incertaine. On peut toutefois mentionner un probable " coin à fendre ": le côté mâchuré est opposé à un tranchant portant de multiples esquillements d'utilisation.

\section{Les outils percutants}

73 Les percuteurs (6 pièces) sont tous des objets globuleux en silex, dont au moins la moitié de la surface est bouchardée. Il est possible qu'il s'agisse de réutilisations de nucléus, pour au moins deux d'entre eux. Ce sont des objets relativement volumineux et lourds (poids variant entre $390 \mathrm{~g}$ et $512 \mathrm{~g}$, avec une moyenne de $428 \mathrm{~g}$ ). Ces caractéristiques peuvent correspondre à une utilisation pour le débitage des plus grands éclats présents dans la série.

Douze nucléus portent des surfaces bouchardées de moindre importance, reflétant une utilisation plus ponctuelle.

75 Les bouchardes sphériques (3 pièces) sont des objets en silex façonnées par bouchardage. La quasi-totalité de leur surface est piquetée, aboutissant à une forme sphérique régulière. Leur poids est de $211 \mathrm{~g}, 544 \mathrm{~g}$ et $566 \mathrm{~g}$. La fonction de ces pièces reste incertaine (broyeurs?).

\section{Bilan de l'outillage}

Avec 185 objets, l'outillage du Mesnil-Esnard constitue un ensemble représentatif statistiquement acceptable. L'outillage retouché représente $6,8 \%$ de la série et 
l'outillage au sens large (intégrant les outils a posteriori) s'élève à 9,3\%. À l'exception de quelques rares objets exogènes, les outils exploitent les éclats souvent corticaux et les quelques éclats laminaires produits sur le site.

La catégorie des grattoirs domine très largement, avec une représentation de 40,5\%. Viennent ensuite les outils a posteriori, qui s'élèvent à $26,5 \%$. La troisième catégorie rassemble les microdenticulés et les pièces apparentées (" éclats micro-esquillés ») totalisant $8,1 \%$. Mais la reconnaissance fonctionnelle de cette dernière classe d'objets repose ici uniquement sur une observation macroscopique qui devrait être validée par une étude tracéologique à fort grossissement. Dans cette attente, il faut retenir que la part des microdenticulés typiques ne représente que $2,7 \%$ des outils.

Parmi les autres catégories, seules les pièces à retouche latérale $(10,2 \%)$ et les denticulés $(7,6 \%)$ forment des lots représentatifs. On trouve ensuite les couteaux à dos et pièces apparentées ( $1,6 \%$ à $3,2 \%$, selon le type considéré), puis les catégories représentées par des individus uniques : racloir à encoche, " couteau quadrangulaire ", éclat à coche, bitroncature, perçoir, ciseau, hache polie et meule en silex $(0,5 \%)$. Parmi ces catégories discrètes, les outils pouvant être rapportés à la fonction de « couteau à moissonner " (racloir à encoche, couteau quadrangulaire et couteau à dos aminci) totalisent $2,6 \%$ de l'assemblage.

\section{Approche chronoculturelle}

La série lithique du Mesnil-Esnard peut être spontanément attribuée à l'horizon chronologique du $\mathrm{III}^{\mathrm{e}}$ millénaire avant $\mathrm{J}$.-C., grâce à la présence dans l'outillage des pièces caractéristiques que sont les microdenticulés et le racloir à encoche. D'autres outils apparentés au couteau à moissonner (couteau à dos aminci, couteau quadrangulaire) se rattachent indubitablement à l'horizon culturel du Néolithique récent et final.

Le débitage d'éclats, quasiment exclusif, ne vient pas contredire cette attribution. L'absence du débitage laminaire est une caractéristique incontournable de la série.

81 À Bettencourt-Saint-Ouen, le débitage laminaire est mal représenté et les microdenticulés sont présents à hauteur de $25 \%$ (Martin et al., 1996). Les datations isotopiques obtenues sur ce site placent l'occupation entre 2450 et $2050 \mathrm{BC}$. En tenant compte du modèle d'une décroissance de la proportion du débitage laminaire à partir du début du III millénaire, il serait tentant de voir ici un indice de rajeunissement de la série du Mesnil-Esnard. Ceci pourrait être conforté par la faible proportion de microdenticulés. Dans la moitié nord de la France, ces objets semblent disparaître totalement à l'âge du Bronze, sauf à Tatihou (Martial, 1995; Ghesquière et al., 1997). S'ils disparaissaient aussi en Haute-Normandie, il semblerait difficile de proposer une datation plus basse que l'horizon du Bronze ancien.

Le racloir à encoche, le couteau quadrangulaire et les couteaux à dos aminci forment une famille d'outils proches sur le plan morphologique et probablement fonctionnel. Leur proportion est faible au sein de l'assemblage (2,6\% au total, avec un unique racloir à encoche, soit $0,5 \%$ pour ce type précis), mais on remarquera qu'à Bettencourt-SaintOuen, la proportion de racloirs à encoche n'est guère plus élevée (4\%). Dans les ensembles 4 à 7 de Poses "Le Vivier/Le Clos Saint-Quentin ", étalés sur un intervalle allant du Néolithique final au Bronze ancien (avec des éléments campaniformes), ces outils sont absents mais le mobilier lithique est indigent dans ces ensembles (Billard et 
al., 1994). E. Martial constate que dans le Nord de la France, les racloirs à encoche sont absents à l'âge du Bronze et inconnus sur ceux de l'Artenac et du Gord (Martial, 1995). On ne peut donc pas, a priori, prendre en compte la proportion de ces objets pour affiner une attribution chronologique, mais il semble tout de même possible d'exclure l'âge du Bronze.

\section{Les vestiges céramiques}

Les tessons découverts au sein de la nappe, déjà peu abondants, sont généralement mal conservés et très fragmentés (aucun profil complet et aucun remontage possible). On dénombre 2 bords, 163 éléments de panses, 6 fonds et 1 élément de préhension (anse en ruban; fig. 15). Deux bords sont attribuables à des formes basses ouvertes. Un autre, accompagné de son col, appartient à une forme haute fermée. Tous les fonds sont plats sauf un qui est légèrement arrondi. Enfin, nous pouvons y ajouter le profil semi complet, appartenant à une céramique grossière de forme haute et à fond plat, trouvé lors du diagnostic.

Les pâtes sont généralement de teintes claires en surface (rouges ou orangées) et sombres à cœur. Quelques pâtes brunes, à cœur noir, ont été cuites en atmosphère réductrice. Les inclusions sont minérales avec majoritairement du silex associé à du quartz et des micas. Seuls deux individus contenaient de la chamotte. Enfin, le mode de montage et le traitement des surfaces sont impossibles à déterminer. 
Figure 15 : Mesnil-Esnard "Les Hautes Haies ", mobilier céramique. Figure 15 : Mesnil-Esnard "Les Hautes Haies", pottery.

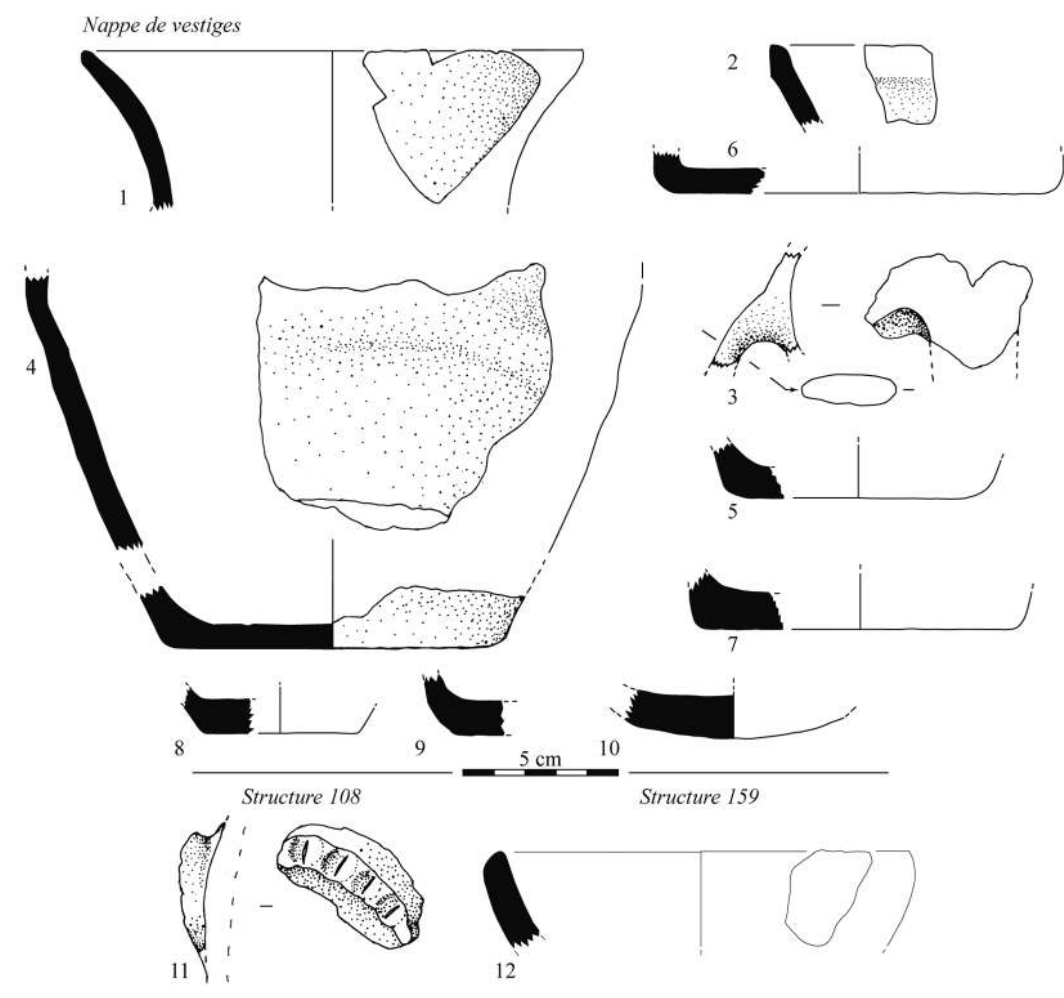

1, 2 et 6 : éléments néolithiques ou protohistoriques ; 3 à 5 et 7 à 12 : éléments de la fin du IIIe millénaire av. J.-C.

1, 2 and 6: Neolithic or Protohistoric pottery; 3 to 5 and 7 to 12: potteries of the end of the 3rd millennium BC. Dessin J.-Y. Noël.

Une attribution typochronologique globale est difficile à arrêter pour ce petit ensemble mal conservé, issu d'un contexte archéologique peu fiable. Nous ne pouvons exclure la possibilité de mélanges au sein de cette nappe. En effet, considéré individuellement, chaque élément permet de couvrir à la fois le Néolithique moyen comme le montre le probable col de bouteille (fig. $15, \mathrm{n}^{\circ} 1$ ) et toute la protohistoire, comme l'indique les fonds plats et le petit bord anguleux de l'individu 2 (fig. 15, $\mathrm{n}^{\circ} 2$; Fromont et al., 2006). Cependant, nous notons une nette majorité de pâtes claires, grossières dans lesquelles de la chamotte a ponctuellement été observée. Ces caractéristiques technologiques associées à des fonds plats irréguliers (épaissis ou arrondis) et à une anse en ruban, ne seraient pas incohérents dans un corpus de la fin du $\mathrm{III}^{\mathrm{e}}$ ou du début du $\mathrm{II}^{\mathrm{e}}$ millénaire avant J.-C.

\section{Les structures excavées (nature et mobilier)}

Les structures excavées ne sont pas très nombreuses. Elles ne trahissent aucune organisation de l'espace fouillé et ont rarement livré un mobilier susceptible de les dater. Leur relation avec la nappe de silex ne peut donc être appréhendée. Seule la structure 21, par sa position, pourrait lui être associée. Nous présenterons donc succinctement les principales d'entre elles. Nous ne détaillerons pas les fossés récents qui matérialisent le reste d'une organisation parcellaire. 
Deux structures plutôt bien conservées, $\mathrm{n}^{\circ} 108$ et 147 , destinées à l'usage du feu, ont été fouillées dans la partie ouest du décapage (fig. 3 et 16). Quasiment similaires, elles sont constituées de deux creusements circulaires à ovoïdes, réunis par un court couloir. La fosse d'accès s'illustre par l'abondance des charbons dans le remplissage alors que la chambre de chauffe est marquée par une zone de limon rubéfié. Ce type de structure s'apparente aux fours à sole suspendue particulièrement fréquents durant la protohistoire.

Figure 16: Mesnil-Esnard «Les Hautes Haies » : le four à sole suspendue 147. Figure 16 : Mesnil-Esnard "Les Hautes Haies": oven 147.

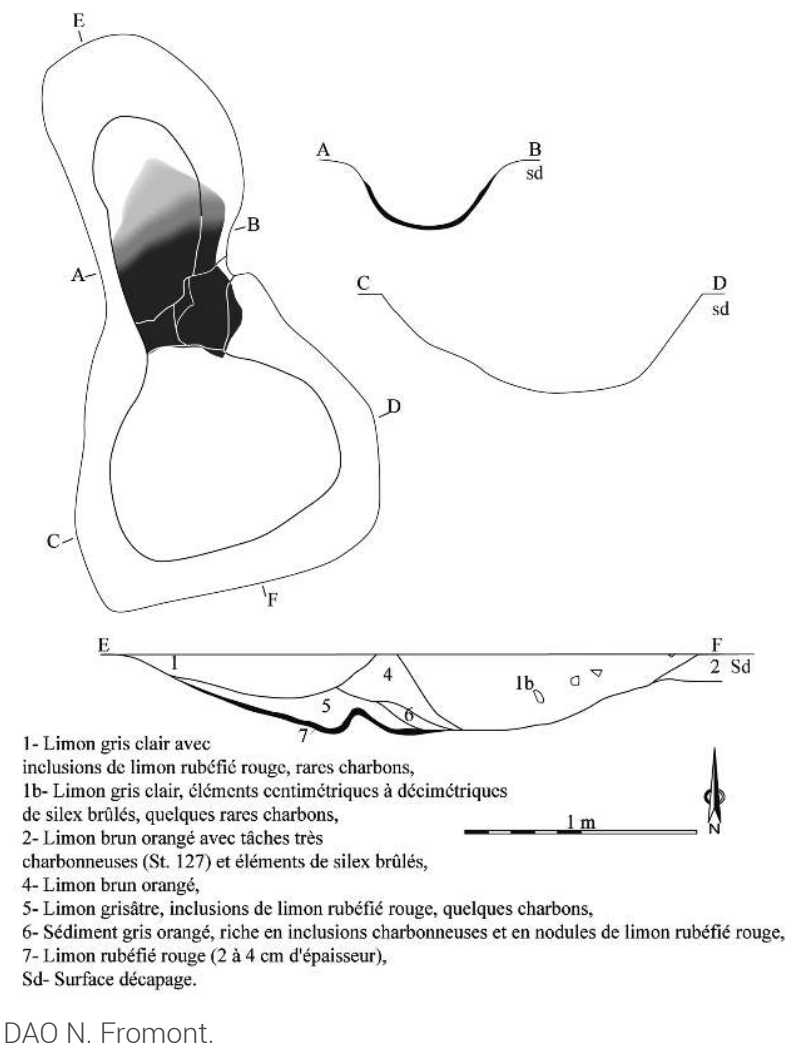

Six fragments de panse et quelques éclats de silex sont issus du four 147. Le four 108 a livré deux tessons de panse ainsi qu'un élément de préhension consistant en un cordon arciforme décoré d'impressions à l'ongle (fig. 15, $\mathrm{n}^{\circ} 11$ ). Il est habituellement attribué au Groupe des urnes à décor plastique, inclus dans le complexe "Manche - Mer du Nord" et caractériserait l'âge du Bronze ancien. Dans la région, les plus anciens cordons arciformes sont attribués à la fin du $\mathrm{III}^{\mathrm{e}}$ millénaire, en contexte Néolithique final/Bronze ancien à Saint-Vigor-d'Ymonville (Marcigny et al., 2002). Sur le site de Poses «Le Vivier/Le Clos Saint-Quentin », les deux cordons arciformes sont attribués à un horizon Bronze ancien étroitement lié au complexe campaniforme bien présent sur le site (Billard et al., 1994). De la même manière, à Grossœuvre "Viancourt », le cordon arciforme est accompagné de formes attribuées à la céramique commune campaniforme (Billard et Paez-Rezende, 2000). À Bolbec, une urne à cordon digité est calée dans le Bronze moyen (Aubry et al., 1994). Un vase possédant ce décor associé à un cordon horizontal encoché trouvé aux " Fondriaux » à Bernières-sur-Seine est attribué au Bronze final (Paez-Rezende, 1998), attribution proposée par comparaison avec les 
exemplaires du groupe d'Eramecourt. Finalement, la datation de la structure 108 reste donc imprécise, mais pourrait être envisagée pour la première moitié de l'âge du Bronze.

L'utilisation du feu est également attestée sous deux autres formes : un probable foyer $\left(n^{\circ} 104\right)$ et une zone de rejets cendreux $\left(n^{\circ} 127\right)$. Le foyer est représenté par une zone où le sédiment est enrichi de nodules de limon rubéfié. Il n'y a pas, à proprement parler, de sole. La structure 127 est une tache charbonneuse au sein du sédiment limoneux, aucun creusement n'ayant été mis en évidence. Seule la pénétration, par bioturbation, des particules charbonneuses donne de la profondeur à la structure.

Découverte et partiellement explorée lors du diagnostic, une fosse profonde $\left(\mathrm{n}^{\circ} 6 / 181\right)$, implantée dans la partie ouest du décapage, est interprétée comme un silo (fig. 17 ; Aubry et al., 2004). Aucun mobilier n'y a été découvert. Le creusement, aux parois légèrement en sape, mesure 85 centimètres de profondeur pour une longueur de 130 centimètres et une largeur de 100 centimètres. La forme à l'ouverture ainsi que la différence de hauteur $\mathrm{du}$ fond suggèrent un creusement en plusieurs temps. L'hypothèse de plusieurs phases d'utilisation semble confirmée par l'organisation du comblement limoneux. Il comporte des charbons de bois et des nodules de terre rubéfiée qui pourraient provenir de la plage de sédiment rubéfié située à proximité immédiate (structure 104).

Figure 17 : Mesnil-Esnard "Les Hautes Haies » : la fosse silo 6/181.

Figure 17: Mesnil-Esnard "Les Hautes Haies": silo 6/181.

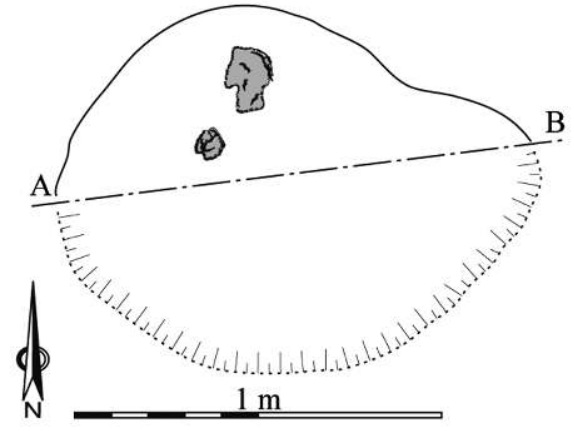

1- limon argileux brun gris assez homogène, quelques charbons, nodules de terre cuite et blocs de silex,

2- limon gris marbré très hydromorphe, rares charbons, nombreux nodules de terre cuite,

3- Amalgame de 4 et de 2 ,

4- Limon argileux brun orangé et

très rares charbons,

5- Limon argileux orangé et oxydé avec inclusions hydromorphes,

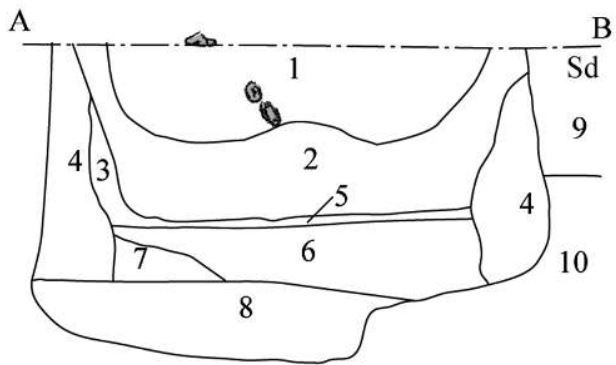

6- Limon argileux gris marbré très hydromorphe (2), rares charbons et très nombreux nodules de terre cuite,

7- Identique à 3 en moins oxydé, 8- Limon argileux hydromorphe à matrice brune avec très nombreuses inclusions de charbons et de terre cuite, 9- Argile hydromorphe et limon brun orangé, 10- Limon argilo-sableux brun jaune, Sd- Surface décapage.

DAO B. Aubry.

Également découverte lors du diagnostic, la fosse 21 a livré un ensemble constitué de deux meules dormantes et d'un broyon appareillé à l'une d'entre elles (fig. 18, op.cit.). Le remplissage est constitué de limon brun qui, sous le dépôt de meules, est enrichi en inclusions de limon rubéfié. Le mobilier est uniquement représenté par le dépôt d'outils de mouture confectionnés dans deux faciès similaires de grès (fig. 18). 
Figure 18 : Mesnil-Esnard "Les Hautes Haies » : la fosse 21 et son dépôt d'outils de mouture. Figure 18 : Mesnil-Esnard "Les Hautes Haies": pit and grinding tools.

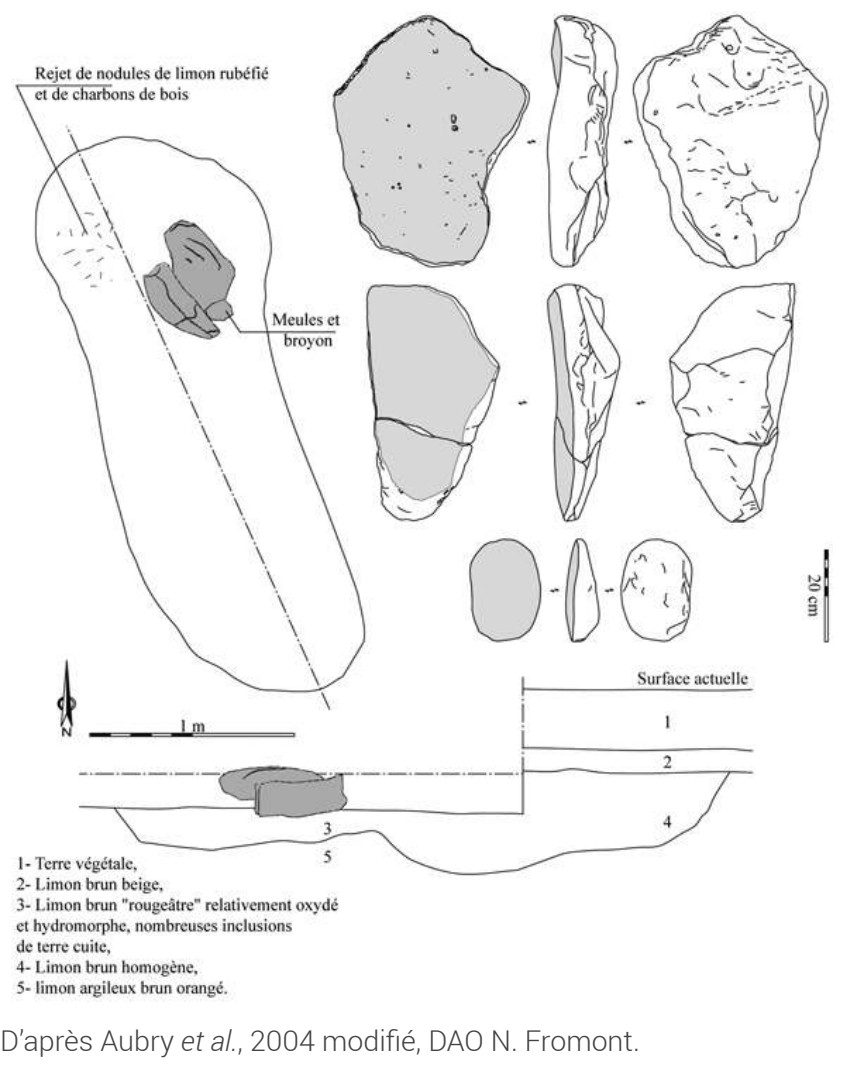

Le premier objet est une grande meule fortement concave aménagée dans un bloc très large. La partie active occupe toute la surface d'une des grandes faces. Sa surface piquetée est encore perceptible malgré un émoussé poussé et des zones usées et polies. Le dos de la meule correspond à une surface naturelle et seuls quelques secteurs de la tranche portent des traces d'aménagement sous la forme d'enlèvements abrupts et peu couvrants. La seconde meule est aménagée sur un bloc de grès allongé. La surface active est légèrement plus réduite que celle de la face du bloc. Les zones polies y sont beaucoup plus rares et la surface paraît, dans son ensemble, moins usée que précédemment. Les « cratères » créés par le piquetage sont plus marqués. Le dos de la pièce porte de nombreux enlèvements obtenus à partir d'une nervure. D'autres, perceptibles sur la tranche, sont obtenus à partir de la face portant la surface active.

Enfin, la molette est représentée par un bloc ovalaire. Une des faces, régulièrement concave, porte des traces de piquetage plus ou moins émoussées et parfois même polies. Le dos de la pièce correspond à une surface naturelle simplement aménagée par quelques enlèvements.

Sur les sites néolithiques et protohistoriques, les outils de mouture sont fréquemment représentés par des fragments en position secondaire (abandon après cassure ou en « fin de vie » de l'outil). Plus rares sont les pièces entières organisées en dépôt. Une récente étude portant sur les dépôts d'outils de mouture en contexte néolithique ancien, conduit à envisager l'hypothèse qu'ils pourraient constituer « des rangements provisoires d'outils dont l'entretien serait en cours et inachevé » (Hamon et Samzun, 2004, p. 20). Avec la structure 21 du Mesnil-Esnard, nous sommes peut-être dans un cas de figure équivalent. 
La structure 105 est apparue très haut lors du décapage, du fait de la présence d'un petit lot de vestiges contenu dans du limon. Ses contours sont flous. Elle a livré trentesix produits de débitage, trois nucléus et cinq outils. Ces derniers sont représentés par trois denticulés, un éclat retouché et un perçoir. Des remontages ont pu être effectués, notamment, plusieurs éclats et un denticulé qui se raccordent à un même nucléus. Il est remarquable de constater l'homogénéité et la contemporanéité de cet assemblage lithique dans la structure. Ces éléments illustrent une activité ponctuelle de débitage en relation avec la structure, en vue de produire des outils liés à une activité indéterminée qui a requis l'utilisation d'au moins trois types d'outils retouchés différents. S'y ajoutent quatre fragments de panses façonnés dans des pâtes siliceuses cuites en atmosphère partiellement oxydante.

\section{Conclusion}

Pour l'essentiel, les vestiges retrouvés aux « Haute-Haies » sont issus d'activités liées à la production et à l'utilisation d'objets en silex. Il n'a pu être déterminé s'ils sont en position primaire (amas de débitage par exemple, pièces abandonnées sur « un niveau de sol/circulation ») ou dérivée (rejet de déchets). L'outillage, relativement abondant, est varié, ce qui pourrait conduire à envisager qu'il découle d'activités domestiques peut-être en lien avec certaines structures excavées découvertes sur le site. Cette zone pourrait alors s'intégrer dans une entité plus vaste de type habitat ou village. À moins qu'elle ne constitue une "annexe" au sein du territoire exploité par un groupe d'individus. Dans les deux cas, des décapages plus vastes autour de ces nappes de mobilier auraient été bénéfiques à leur compréhension.

Globalement, l'absence ou la rareté de vestiges de nature autre que l'industrie en silex complique l'analyse tant fonctionnelle que chronoculturelle de l'occupation. Ainsi, il faut prendre avec précaution la datation proposée à partir du mobilier céramique : entre le Néolithique final et l'âge du Bronze. Un faisceau d'indices oriente clairement la série lithique du Mesnil-Esnard vers un horizon chronologique similaire, soit vraisemblablement durant le $\mathrm{III}^{\mathrm{e}}$ millénaire avant J.-C. Plusieurs types d'outils permettent d'écarter la possibilité d'un ensemble plus tardif. De nombreuses questions restent soulevées, notamment sur la fonction de certains objets, en l'absence d'analyse tracéologique. On insistera sur cet aspect, car cette analyse fonctionnelle peut s'avérer significative pour l'approche chronoculturelle. L'étude typologique a toutefois livré des éléments particulièrement intéressants, comme les pièces à dos aminci et fracture distale ("couteau à dos aminci »). Avec également un nouveau "couteau quadrangulaire ", la série du Mesnil-Esnard vient valider ce type de pièces initialement identifiées sur d'autres sites (Martin et al., 1996; Martinez et al., 1999). L'approche tracéologique permettrait également de mieux appréhender l'origine de ces nappes de silex taillés.

98 L'assemblage lithique du Mesnil-Esnard vient donc poser un nouveau jalon pour l'industrie lithique de la fin du Néolithique en Haute-Normandie. Il est aujourd'hui difficile d'être plus précis, mais gageons que de nouvelles séries viendront à l'avenir enrichir et favoriser les comparaisons, permettant alors d'affiner l'attribution chronoculturelle ici proposée. 


\section{BIBLIOGRAPHIE}

AUBRY B., PAEZ-REZENDE L. et VERDELET F., 1994 - Bolbec, déviation, Document final de synthèse, Association pour les fouilles archéologiques nationales, Service régional de l'archéologie de Haute-Normandie, Rouen; non paginé.

AUBRY B., BRETON D. et LEPINAY D., 2004 - Mesnil-Esnard «Le Chemin des Ondes », Document final de synthèse, Institut national de recherches archéologiques préventives, Service régional de l'archéologie de Haute-Normandie, Le Grand-Quevilly, p. 8.

BILLARD C., BOURHIS J.-R., DESFOSSÉS Y., ÉVIN J., HUAULT M.-F., LEFEBVRE D. et PAULET-LOCARD M.-A., 1991 «L'habitat des "Florentins" à Val-de-Reuil (Eure) », in BILLARD c. (dir.), « Nouveaux sites campaniformes de la basse vallée de la Seine », Gallia Préhistoire, 33, p. 140-171.

BILLARD C., AUBRY B., BLANCQUAERT G., BOURHIS J.-R., HABASQUE G., MARINVAL P., PINEL C. et ROPARS A., 1994 «Poses "Le Vivier/Le Clos-Saint-Quentin" (Eure), l'occupation de la plaine inondable au Néolithique et au début de l'âge du Bronze », Revue archéologique de l'Ouest, 11, p. 53-113.

BILLARD C., BLANCHET J.-C. et TALON M., 1996 - « Origine et composantes de l'âge du Bronze ancien dans le Nord-Ouest de la France ", in moRdANT c. et GAIFFE o. (dir.), Cultures et sociétés du Bronze ancien en Europe, $117^{\mathrm{e}}$ congrès national des Sociétés historiques et scientifiques, Clermont-Ferrand, 1992, Pré- et Protohistoire, édition du Comité des travaux historiques et scientifiques, Paris, p. 579-601.

BILLARD C. et PAEZ-REZENDE L., 2000 - « Un habitat du Bronze ancien à Grossœuvre "Viancourt" (Eure) », Bulletin de la Société préhistorique française, 97, 2, p. 275-289.

BRUNET P., COTTIAUX R., HAMON T., LANGRY-FRANÇOIS F., MAGNE P. et SALANOVA L., 2005 - « La céramique de la fin $d u I^{e}$ et III millénaire dans le Centre-Nord de la France. Bilan documentaire ", in VAN DER LINDEN м. et salanova l. (dir.), Le IIIe millénaire dans le Nord de la France et en Belgique, Actes de la journée d'études du 8 mars 2003, Lille, Anthropologica et Praehistorica, Mémoire de la Société préhistorique française, 35, Paris , p. 155-178.

COGUEL P. et SANGNIER J., 1967 - Carte géologique de la France (1/50 000), feuille Rouen-est (XX-11), Bureau des ressources géologiques et minières, Orléans.

COGUEL P. et SANGNIER J., 1967 - Notice explicative, carte géologique de la France (1/50 000), feuille Rouen-est (XX-11), Bureau des ressources géologiques et minières, Orléans, 14.

FROMONT N., JUHEL L., NOËL J.-Y., COUTARD S., THÉRON V., BEUGNET L., CHANTREUIL L., COTTARD A., NÉRÉ É., BIARD M., TESSIER V. et KERROUCHE V., 2006 - Mesnil-Esnard « Les Hautes Haies », Document final de synthèse, Institut national de recherches archéologiques préventives, Service régional de l'archéologie de Haute-Normandie, Rouen, p. 132.

GHESQUIÈRE E., MARCIGNY C., DESLOGES J. et LE GAILLARD L., 1997 - « Le silex à l'âge du Bronze. L'industrie lithique à l'âge du Bronze du site de l'île Tatihou à Saint-Vaast-la-Hougue (Manche) », Revue archéologique de l'Ouest, 14, p. 27-47.

HAMON C. et SAMZUN A., 2004 - « Découverte d'un dépôt de mouture et de broyage daté du Néolithique ancien (culture Villeneuve-Saint-Germain récent, v. 4 700-4 600 avant notre ère), à Saint-Denis 66-70, rue du Landy (Seine-Saint-Denis) ", Bulletin de la Société préhistorique française, 101, 3, p. 611-613.

HONEGGER M., 2011 - L'industrie lithique taillée du Néolithique moyen et final de Suisse, Collection « Recherches archéologiques », CNRS éditions, Paris, p. 356. 
MARCIGNY C., BLANCHET S., BOUJOT C., CARPENTIER V., CLÉMENT-SAULEAU S., CORDE D., DELOZE V., DIETSCH-SELLAMI M.-F., GHESQUIÈRE E., GIAZZON D., GALLOUIN É., HUGOT C., RICHE C., LECLERCQ E., LEPAUMIER H., PROST D. et SELLAMI F., 2002 - Saint-Vigor-d'Ymonville (Seine-Maritime) « Les Sapinettes » et « La Mare des Mares » (Carrière Lafarge, tranches 1 et 2), I (du Néolithique ancien au Néolithique moyen), Document final de synthèse, Institut national de recherches archéologiques préventives, Service régional de l'archéologie de Haute-Normandie, Rouen, p. 152.

MARCIGNY C., GHESQUIÈRE E., CLÉMENT-SAULEAU S., VERNEY A., CHANCEREL A., DESLOGES J., GALLOUIN É., GIAZZON D., GRÉGOIRE V., HUGOT C., JAHIER I., LE GOFF E., LEPAUMIER H., RENAULT V., SAN JUAN G., SAVARY X. et VERRON G., 2005 - « L’âge du Bronze en Basse-Normandie : définition par le mobilier céramique, une première tentative », in BOURGEOIS J. et TALON m. (dir.), L'âge du Bronze du Nord de la France dans son contexte européen, Comité des travaux historiques et scientifiques, Association pour la promotion des recherches sur l'âge du Bronze, Paris, p. 303-332.

MARTIAL E., 1995 - « L'industrie lithique à l'âge du Bronze dans le Nord - Pas-de-Calais. Les exemples de Fréthun et Rœux », Les Cahiers de préhistoire du Nord, 15, Amiens, p. 127.

MARTIN J.-M., MARTINEZ R., PROST D., BOUILLOT J., MATTERNE V., MUNOD A.-V. et PERNAUD J.-M., 1996 Bettencourt-Saint-Ouen (Somme), Document final de synthèse, Association pour les fouilles archéologiques nationales, Service régional de l'archéologie du Nord - Pas-de-Calais, Amiens, 2 vol., $127 \& 306$.

MARTINEZ R., PROST D., BIARD M., BOULAY G. et LÉON G., 1999 - Guerny « Le Bois Madame » (Eure), Déviation RN 14, Document final de synthèse, Association pour les fouilles archéologiques nationales, Service régional de l'archéologie de Haute-Normandie, Rouen, non paginé.

PAEZ-REZENDE L., 1998 - Extension de carrière, commune de Bernières-sur-Seine « Les Fondriaux » (Eure), Document final de synthèse, Association pour les fouilles archéologiques nationales, Service régional de l'archéologie de Haute-Normandie, Rouen, p. 65.

PROST D., 1999 - Bernières-sur-Seine « La Banque » (Eure), site C, Document final de synthèse, Association pour les fouilles archéologiques nationales, Service régional de l'archéologie de Haute-Normandie, Rouen, non paginé.

SALANOVA L., 2000 - La question du Campaniforme en France et dans les îles Anglo-Normandes, production, chronologie et rôle d'un standard céramique, Comité des travaux historiques et scientifiques, Société préhistorique française, Paris, p. 392.

SALANOVA L., AUGEREAU A., BOUDUET L., BRUNET P., COSTA L., COTTIAUX R., COUDERC J., GOUTELARD A., HAMON T., IHUEL I., JAMET E., LANGRY-FRANÇOIS F., LOUBOUTIN C., MAGNE P., MAINGAUD A., MALLET N., MARTINEAU R., MILLE B., MILLET-RICHARD L.-A., POLLONI A., RENARD C., RICHARD G., SAMZUN A., SIDÉRA I. et SOHN M., 2003 - Le

III millénaire avant J.-C. dans le Centre-Nord de la France : définition et interactions des groupes culturels, Bilan de trois années de recherche, $147 \mathrm{p}$.

SALANOVA L. et VAN DER LINDEN M., 2004 - « La fin du Néolithique dans le Nord de la France et en Belgique : bilan et perspectives », in VAN DER LINDEN M. et SALANOVA L. (dir.), Le IIIe millénaire dans le Nord de la France et en Belgique, Actes de la journée d'études du 8 mars 2003, Lille, Anthropologica et Praehistorica, Mémoire de la Société préhistorique française, 35, Paris, p. 225-226.

STRAHM C., 2004 - « Le phénomène campaniforme et les composantes autochtones non campaniformes », Bulletin de la Société préhistorique française, 101, 2, p. 201-206.

WATTÉ J.-P., 1992 - Le Néolithique en Seine-Maritime, suppl. Bulletin trimestriel de la Société géologique de Normandie et des Amis du muséum du Havre, 77, 2 : 3 vol., 797. 
WATTÉ J.-P., 2004 - « À propos des sources de matières premières utilisables et utilisées par les préhistoriques en Seine-Maritime : le silex cénomanien, un bon marqueur pour la mise en évidence du transport de matières premières et d'objets finis ", Haute-Normandie archéologique, t. 9, 2004, p. 13-35.

\section{RÉSUMÉS}

La fouille du site des « Hautes Haies » au Mesnil-Esnard a conduit à la découverte d'une nappe de vestiges, essentiellement lithiques, conservée sous les labours. L'étude technologique et typologique de ce mobilier envisage une attribution de l'occupation au Néolithique final/Bronze ancien. Elle met également en évidence des singularités dans l'outillage qui rapproche la série d'autres issues du Nord-Ouest de la France et appuie sur la nécessité d'une approche tracéologique pour dépasser les limites de la typologie. Cette approche conduirait également à mieux cerner les activités qui ont généré les vestiges abandonnés sur le site, lesquelles restent difficiles à déterminer. Quelques structures en creux ont également été découvertes. Leur datation et relation avec la nappe de vestiges ne peuvent être précisément établies.

The excavation in Mesnil-Esnard "Les Hautes Haies" has led to the discovery of a layer of mainly lithic objects (débitage) dating from the Final Neolithic/Early Bronze Age preserved under the topsoil. The study has revealed specificities of the tools which links this series to others from sites in the Northwest of France and underlines the necessity of a traceological study which should yield better results than a simple typological approach. It would also contribute in determining the site's function, which remains unknown. Some excavated features were also discovered. Their date and link to the layer of lithic artifacts cannot as yet be exactly established.

La excavación del sitio "Les Hautes-Haies" en Mesnil-Esnard llevó al descubrimiento de una capa de ruinas, en su mayoría líticas, mantenidas en el arado. El estudio tecnológico y tipológico de este mobiliario deja pensar a una ocupación durante el Neolítico final/Bronce antiguo. También permite poner de relieve las peculiaridades de la herramienta, que se parece a otras series del noroeste de Francia, y demuestra la necesidad de un enfoque traceológico para superar las limitaciones de la tipología. Este enfoque también podría conducir a una mejor comprensión de las actividades que generaron los restos que quedan en el sitio, a pesar de que quedan difíciles de determinar. Algunas estructuras en forma de hueco también se han descubierto. Su datación y la relación con la capa de ruinas no pueden establecerse con precisión.

\section{INDEX}

Palabras claves : finales del Neolítico / Edad del Bronce, capa de ruinas, mobiliario lítico, horno, silo

Keywords : Final Neolithic, Bronze age, debitage products, tools, oven, pit, silo

Mots-clés : nappe de mobilier, four, fosse, Néolithique, mobilier

\section{AUTEURS}

\section{NICOLAS FROMONT}

Inrap Pays-de-la-Loire, 4 rue du Tertre, 44477 CARQUEFOU - nicolas.fromont@inrap.fr 


\section{LAURENT JUHEL}

Inrap Bretagne, 37 rue du Bignon CS 67737, 35577 Cesson-Sévigné - laurent.juhel@inrap.fr JEAN-YVES NOËL

Conseil général d'Eure-et-Loir - Service archéologie, allée du général-Martial-Valin, 28000 Chartres - jean-yves.noel@cg28.fr

\section{BRUNO AUBRY}

Inrap Haute-Normandie, 30 boulevard de Verdun, Immeuble Jean-Mermoz, 76120 Le GrandQuevilly - bruno.aubry@inrap.fr 